\title{
Ranges of $\mathrm{B}, \mathrm{Cd}, \mathrm{Cr}, \mathrm{Cu}, \mathrm{Fe}, \mathrm{Pb}, \mathrm{Sr}, \mathrm{Tl}$, and $\mathrm{Zn}$ Concentrations and Isotope Ratios in Environmental Matrices from an Urban Area
}

\author{
Nicola Pallavicini $\mathbb{D}^{1,2}$ Emma Engström, ${ }^{1,2}$ Douglas C. Baxter, ${ }^{2}$ Björn Öhlander, ${ }^{1}$ \\ Johan Ingri, ${ }^{1}$ Scott Hawley, ${ }^{3}$ Catherine Hirst, ${ }^{4}$ Katerina Rodushkina, ${ }^{5}$ \\ and Ilia Rodushkin $\mathbb{D I D}^{1,2}$ \\ ${ }^{1}$ Division of Geosciences and Environmental Engineering, Luleå University of Technology, S-971 87 Luleå, Sweden \\ ${ }^{2}$ ALS Laboratory Group, ALS Scandinavia AB, Aurorum 10, S-977 75 Luleă, Sweden \\ ${ }^{3}$ Department of Earth Sciences, Durham University, Durham, UK \\ ${ }^{4}$ Department of Geosciences, Natural History Museum, Stockholm, Sweden \\ ${ }^{5}$ Department of Chemistry, Uppsala University, Uppsala, Sweden
}

Correspondence should be addressed to Nicola Pallavicini; nicola.pallavicini@alsglobal.com

Received 2 June 2018; Accepted 18 November 2018; Published 16 December 2018

Academic Editor: Pedro D. Vaz

Copyright (c) 2018 Nicola Pallavicini et al. This is an open access article distributed under the Creative Commons Attribution License, which permits unrestricted use, distribution, and reproduction in any medium, provided the original work is properly cited.

\begin{abstract}
Isotopic information may provide powerful insight into the elemental cycling processes which occur in natural compartments. Further implementation of isotopic techniques in natural sciences requires a better understanding of the range of elemental and isotopic compositional variability in environmental matrices. This study assesses the local-scale concentration and isotopic composition variability of nine elements: boron $(\mathrm{B})$, cadmium $(\mathrm{Cd})$, chromium $(\mathrm{Cr})$, copper $(\mathrm{Cu})$, iron $(\mathrm{Fe})$, lead $(\mathrm{Pb})$, strontium $(\mathrm{Sr})$, thallium $(\mathrm{Tl})$, and zinc $(\mathrm{Zn})$ in lysimetric waters, mushrooms, litter, needles, leaves, and lichens. Sequential extractions were also performed on soil samples from 6 depth profiles providing more detailed information on the variability of elemental concentrations and isotope ratios between the elemental pools present in soil. For most of the sample types studied the range of isotopic variability between samples spans almost the entire ranges reported in the literature for natural samples. These results represent a starting point for discussing the role of natural variability in isotopic studies (for example, as a limiting factor in the use of isotopic mixing models) and a baseline for future in-depth studies examining the controls on isotope fraction in natural systems.
\end{abstract}

\section{Introduction}

The development of isotope-based techniques in environmental science has grown exponentially in the last few decades [1-6]. Recent studies in the field are increasingly adopting multielemental approaches to determine sample provenance [7-9], fingerprint pollution sources [10-14] and identify biochemical processes $[15,16]$. Ultimately, the usefulness of all such single and multielemental isotope methods in biological and environmental sciences is limited by the uncertainties of interpreting the isotope ratio data [17].
Historically, analytical (im)precision was a significant barrier to unambiguous data interpretation for stable $[18,19]$ and even radiogenic isotopic systems [20]. The advent and continued development of multiple collector inductively coupled plasma mass spectrometry (MC-ICPMS) has greatly improved analytic precision attainable. Nowadays, the ability to unambiguously interpret highquality MC-ICP-MS data is increasingly limited by a lack of data on the isotopic variability of environmental samples, i.e., is the identified difference in isotopic compositions between two samples within a range to be expected for the sample type given natural variability or not? 
The severity of the issue was highlighted in a previous study whereof the total concentrations and isotope ratios of a number of elements in leaves, needles, and mushrooms from northern Sweden were determined [2]. Even after removing some known sources of variation such as sampling height (for leaves and needles) and sampling period, very broad ranges in isotopic compositions of many elements were found across samples collected over a confined geographic area. The variability was attributed to a combination of differences in accumulation pathways, soil type, subsoil geology, proximity to local contamination sources, sampling period, and weather preceding sampling occasion [21-23]. Given such a large number of confounding factors may contribute to the isotopic composition of environments samples, it was concluded that while foliage samples may provide highly spatially and temporally resolved snapshots of elemental and isotopic interactions, a better understanding of the individual variables within the system is needed to understand the observed isotopic variability in bioindicators.

Therefore, the aim of this work is to build on the previous work [2] and extend a comprehensive dataset of concentrations and isotopic compositions to nine elements in a variety of environmental samples (topsoil, lysimetric waters, mushrooms, litter, wood ash, needles, leaves, and lichens). As the fate and behaviour of an element in soil is dependent on the physical/chemical form of that element in soils, i.e., dissolved, exchangeable, included in the mineral lattice, or insoluble [24], distinguishing the subpools is an important complement to bulk concentration and isotope ratio measurements. The sequential extraction procedure (SEP) used by this study allowed the determination of 6 functionally defined elemental pools from 6 soil profiles from urban and suburban locations.

The elements measured in this study are currently broadly used across many environmental fields (disciplines) to study a wide range of processes. Several of the elements $(\mathrm{Zn}, \mathrm{Fe}, \mathrm{Cu}$, and $\mathrm{B})$ are essential for plant growth and can be important cofactors in other biological processes. Moreover, the elements included in the study can be of environmental concern $(\mathrm{Cd}, \mathrm{Pb}, \mathrm{Cr}, \mathrm{Cu}, \mathrm{Zn}$, and $\mathrm{Tl}$ ) and/or are commonly used tracers in provenance studies ( $\mathrm{Sr}, \mathrm{B}$, and $\mathrm{Pb}$ ). Overall, both radiogenic ( $\mathrm{Sr}$ and $\mathrm{Pb}$ ) and stable isotopic systems $(\mathrm{B}$, $\mathrm{Cd}, \mathrm{Cr}, \mathrm{Cu}, \mathrm{Fe}, \mathrm{Sr}, \mathrm{Tl}$, and $\mathrm{Zn}$ ) are included and a single multielement/multi-isotopic analytical procedure [2] can be used to measure them all.

The local-scale variability in isotopic composition was compared with the published assessments of global isotopic variability. For elements like Tl, where there is limited published data on the isotopic compositions of environmental samples, the information thus obtained aids gaining knowledge needed for better understanding of $\mathrm{Tl}$ systematics. For other elements, such as Fe, the information offers a framework for considering the relationship between carefully controlled fractionation experiments and real-world samples. In order to illustrate the significance of such variability assessments, a case study is presented using multiisotope data for two landfills contaminated by tailings from Fe production, Fe and $\mathrm{Cu}$ slag, and fly ash.

\section{Materials and Methods}

2.1. Study Site. All samples in this project were collected in and around the city of Luleå, a medium-sized town situated in northern Sweden in the province of Norrbotten (Figure 1). The original village of Luleå dates back to 1649 [25] and has more recently become industrialized including a major steelworks. Samples collected within approximately $5 \mathrm{~km}$ direct distance from local steelworks and harbours will, for the remainder of this study, be referred to as "city" samples. Samples from a broader area, approximately $10 \mathrm{~km}$ from the major local industries, are referred to as "suburb" samples. The suburban locality was relatively recently a seabed (the study area, as well as entire northern part of the Nordic region is rising due to postglacial up-lift almost $9 \mathrm{~mm}$ a year, due to isostatic glacial readjustment [26]) and has been involved in the urbanization process only from the late 1980s.

The local soil consists mainly of clay and silt loam overlying $1.9 \mathrm{Ga}$ granitic bedrock with minor metasedimentary constituents. The suburban location is specifically characterized by a rather homogeneous sandy and well sorted soil with little variations in grain size. The sampling locations in the urban (city) area are till soils characterized by coarse and heterogeneous grain sizes which were formed at the time the land emerged from a sea some 400 years ago.

2.2. Sample Collection and Preparation. The sampling procedures for bioindicator organisms (mushrooms, $n>60$; one pooled litter sample; leaves, $n>200$; needles, $n>40$; lichens, $n>30)$ are reported in Pallavicini et al. [21] and in Rodushkin et al. [2]. Wood ash (originated mainly from local birch wood) was collected from several dedicated recreational fireplaces in Luleå vicinity and pooled into a single sample. Individual soil samples were collected at the end of July 2015 following the same scheme $(n>150$, approximately $10 \mathrm{~g}$ of soil from upper, predominantly organic, 2$3 \mathrm{~cm}$ layer sampled by acid-washed plastic spoon). To supplement the individual soils, samples six soil profiles, two from the city centre and four from the suburban area hereafter referred to as "city" and "suburb" respectively, were also collected. The profiles were collected using a vertical soil core sampler to a maximum sampling depth of $60 \mathrm{~cm}$ (consistent with typical rooting depth for tree species sampled [27]. Once collected, each soil core was divided into four equal $15 \mathrm{~cm}$ horizontal subsamples and stored in $50 \mathrm{~mL}$ polypropylene screw-capped tubes. The soil subsamples does not directly correspond to different soil horizons. In order to compliment the soil samples, pore-water solutions (approximately $250 \mathrm{~mL}$ ) were collected from nine locations in the town using drain-gauge passive-capillary lysimeters $(n=$ 15) installed at $20 \mathrm{~cm}$ depth. Two heavily contaminated soil samples (landfills) from the same region were also added to this study.

Details on sample preparation procedure including chemicals and reagents used, sample digestion and sequential extraction procedure (SEP), analyte/matrix separation, element concentration, and isotopic analyses are fully 


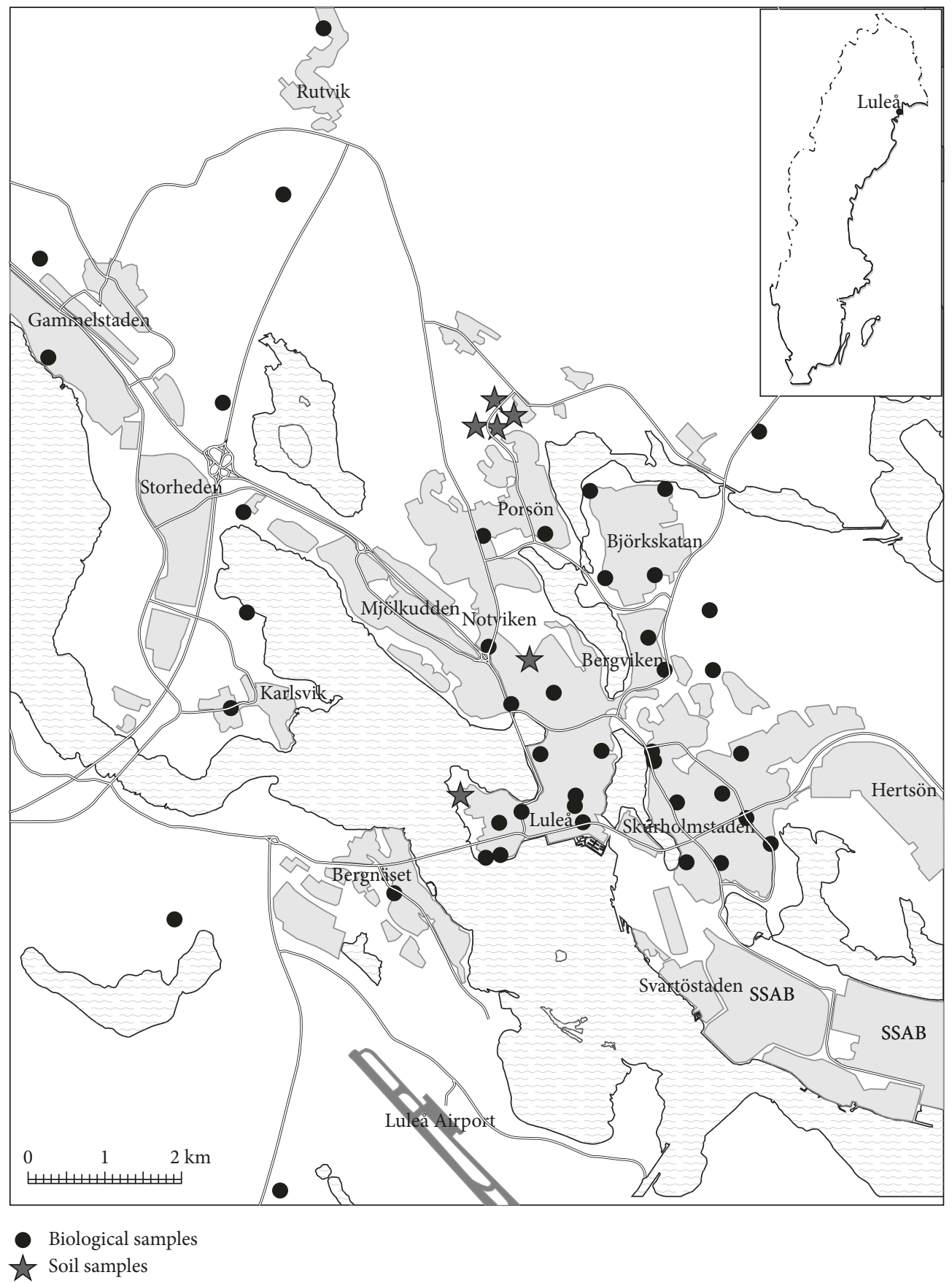

Figure 1: Map of the sampling locations. Filled circles represent the sampling point where biological samples have been collected (leaves, needles, and mushrooms); filled stars represent the locations where soil samples have been collected.

reported in the ESI. The SEP followed a method involving six extractions [28] and has been summarized in Table 1.

2.3. Analytic Performance. Information on operating parameters and measurement conditions for MC-ICP-MS can be found in Table 2 (see ESI for more detailed description). All stable isotopic systems are reported in standard delta $(\delta)$ notation while radiogenic systems are reported as ratios in accordance with standard practices. Stable isotopic differences between samples are reported as capital delta $(\Delta)$ notation denoting the difference between two $\delta$ values.

All solutions were analyzed in duplicate. Signal intensities were transferred to commercially available spreadsheet software for further off-line calculations, including blank, isobaric interference(s) as well as instrumental mass bias corrections. The latter was corrected by the revised exponential correction model [29] using the internal standard (for all isotopic systems but B), and the corrected ratios or $\delta$-values were calculated against bracketing standard solutions. Results from the two 
TAble 1: Sequential extraction scheme.

\begin{tabular}{|c|c|c|c|}
\hline Code & $\begin{array}{l}\text { Extracted } \\
\text { phase }\end{array}$ & Solution & $\begin{array}{l}\text { Volume } \\
(\mathrm{mL})\end{array}$ \\
\hline F1 & Exchangeable & Distilled water & 40 \\
\hline $\mathrm{F} 2$ & Carbonates & $0.11 \mathrm{M} \cdot \mathrm{CH}_{3} \mathrm{COOH}$ (acetic acid) & 40 \\
\hline F3 & Reducible & $\begin{array}{l}0.1 \mathrm{M} \cdot \mathrm{NH}_{2} \mathrm{OH} \cdot \mathrm{HCl} \\
\text { (hydroxylamine hydrochloride) }\end{array}$ & 40 \\
\hline \multirow[t]{2}{*}{$\mathrm{F} 4$} & Oxidizable & $\begin{array}{c}8.8 \mathrm{M} \cdot \mathrm{H}_{2} \mathrm{O}_{2} \text { (hydrogen } \\
\text { peroxide) }\end{array}$ & $10+10$ \\
\hline & & $\begin{array}{c}1 \mathrm{M} \cdot \mathrm{CH}_{3} \mathrm{COONH}_{4}(\text { ammonium } \\
\text { acetate }) \mathrm{pH} 2\end{array}$ & 50 \\
\hline F5 & & Aqua regia & 20 \\
\hline F6 & Residual II & $\mathrm{HF}$ & 10 \\
\hline
\end{tabular}

measurements were used to calculate mean ratios or $\delta$-values and in-run repeatability for each sample.

For the stable isotopic systems, many of the individual isotopes were measured as possible (Table 2) to validate the mass dependent nature of the measurements, but for easy of reporting, only a single $\delta$ value will be used consistently for each system (Table 2 ).

The analytical results for reference standards (QC Loam Soil (VIK) (soil powder) (Eurofins A/S Denmark, Vallensbæk Strand, Denmark), GBW 07410 (soil) (National Research Centre of Geoanalysis, Beijing, China), and IRMM ERM-CC141 (soil powder)) along with typical repeatability and intermediate precision figures for soils, mushrooms, and leaves are reported in Table 3. All reference standards were processed and analyzed in parallel with the samples. It should be noted that results for GBW 07410 and ERMCC141 are calculated using concentrations and isotope ratios for individual sequential extraction fractions and therefore more uncertain. A full list of certified materials used to assess performance of the method for bioindicators is available in the ESI.

Though mass balances for both concentrations (\%-recovery of sum of extracts) and stable isotope ratios (comparison of measured and calculated bulk sample delta values) for all soil samples subject to SEP may aid to accuracy evaluation, this would require thorough homogenization (e.g., by fine grinding) of soils before taking subsample for bulk analyses with accompanying risk of introducing contamination and affecting distribution between phases.

2.3.1. Precision. The intermediate precision for concentration data, defined as the reproducibility of an analytic technique from digestion to measurements on multiple occasions by different analysts, for the reference standards BCR-146R and CRM052 is $<7 \%$ for most elements (Table 3 ). For isotopic ratios, attainable precision varies significantly between isotope systems and matrices studied. It should be stressed that intermediate precision is about two times greater than repeatability (Table 3 ) due to varying element concentrations, slight variations in column yield, efficiency of matrix separation and contamination during column chemistry, instrumental optimization, etc. Intermediate precision, defined in this manner, is therefore considered to provide a valid assessment of the overall method uncertainty or the analytical "resolution" of the procedure. For example, due in large part to low absolute B concentrations, B isotope ratio measurements in mushrooms have a mean uncertainty of approximately $5 \%$ if performed on different occasions, and natural variations in isotopic composition below this level cannot be discerned.

2.3.2. Accuracy. Elemental concentrations in the aqua regia digests were within the $90 \%-110 \%$ range of the certified values for the CRMs except for Sr in CRM052 (Table 3). Reanalysis of the digests by an alternative technique (ICPoptical emission spectrometry) confirmed the doublefocusing sector field ICP-MS (ICP-SFMS) results and the reason for our high reported $\mathrm{Sr}$ concentrations remains unclear. The overall accuracy of the SEP was calculated by summing the elemental concentrations in all the fractions in GBW 07410 and ERM-CC141. The majority of elements were within $10 \%$ of the certified values, but $\mathrm{Cr}$ concentrations samples are around $80 \%$ of the certified values.

The reference materials utilized in this study are not certified for isotopic composition, and, to the authors' knowledge, there is no other published isotope data for the measured CRMs. Therefore, the accuracy of the presented isotope ratio data cannot be directly evaluated. Isotopic information for CRMs presented in Table 3 can aid future interlaboratory calibrations of new reference standards for such matrices.

\section{Results and Discussion}

3.1. Element Concentrations and Isotope Ratios in Environmental Samples. Isotopic composition and concentration data for each element studied is presented in Tables 4-12. The tables are formatted to show the soil profile information for all six SEP fractions in the city and suburb locations (with the reported concentrations representing an average of the individual profiles). For some analytes, only pooled isotopic ratio data is available $(\mathrm{F} 1+\mathrm{F} 2+\mathrm{F} 3$ and $\mathrm{F} 4+\mathrm{F} 5+\mathrm{F} 6)$ due to there being insufficient concentrations in the single extractions to make the isotopic measurements. Concentration and isotopic ratio data for the top soils, lysimetric waters, mushrooms, needles, leaves, lichens, and litter sample are reported, for comparison, below the profile data.

Discussion in this section will start with an examination of multielement patterns which help identify major processes affecting all the samples. The multielement patterns will be followed by a section focused on element-specific patterns including relative differences between the city and suburban samples and relative differences a function of soil depth. Comparing the data between the city and the suburb locations (and between upper and lower soil layers) gives an indication of the isotopic and elemental signature of anthropogenic activity. Depth-related differences in the isotope ratios of labile SEP fractions within the soil profiles might reaffirm a potential role of gradual soil thawing during spring-early summer in the availability of specific element pools with variable isotopic composition to plant root system, as suggested in previous studies [2, 30, 31]. It is important to point out that differences in soil characteristics/ 


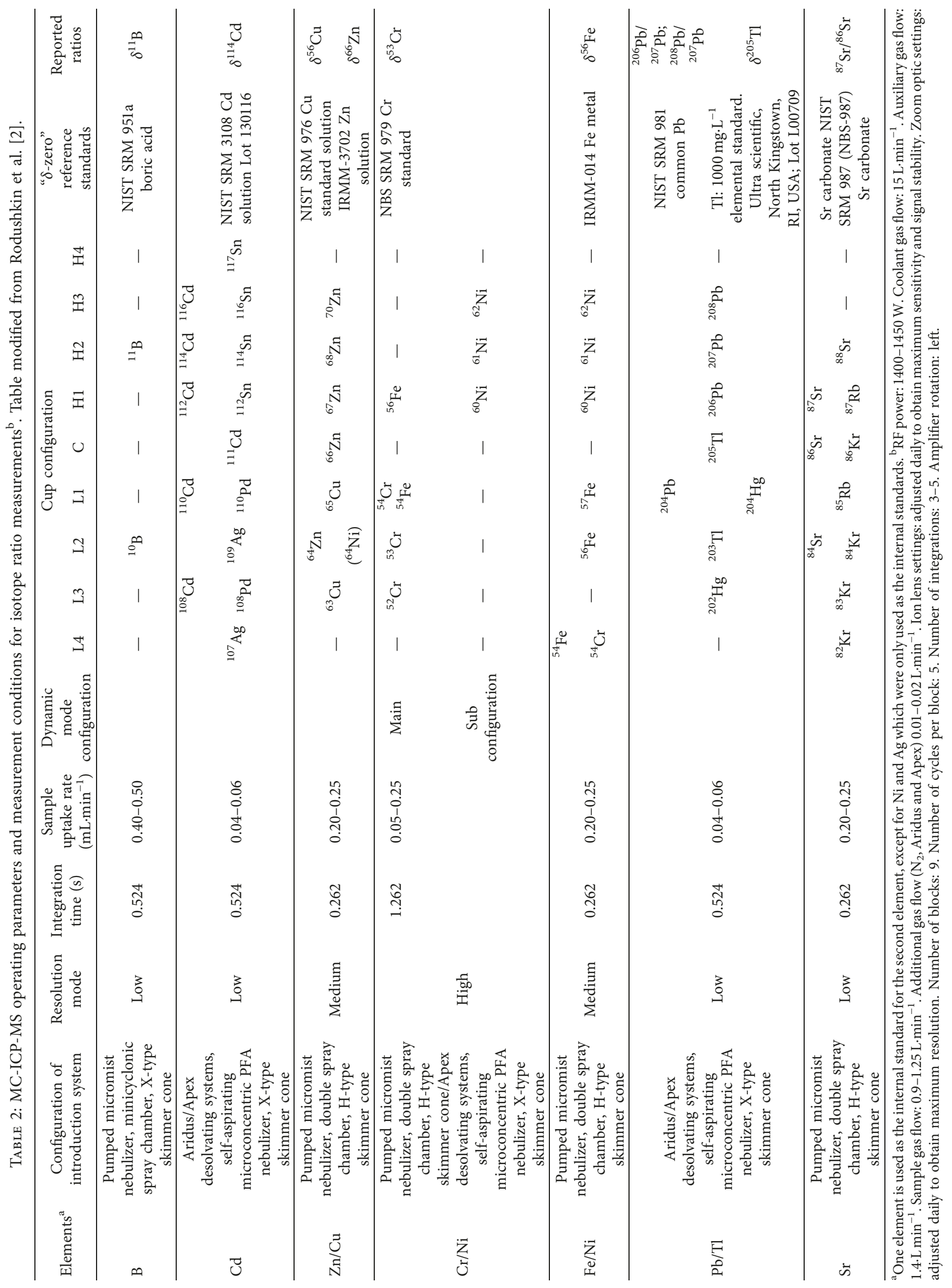




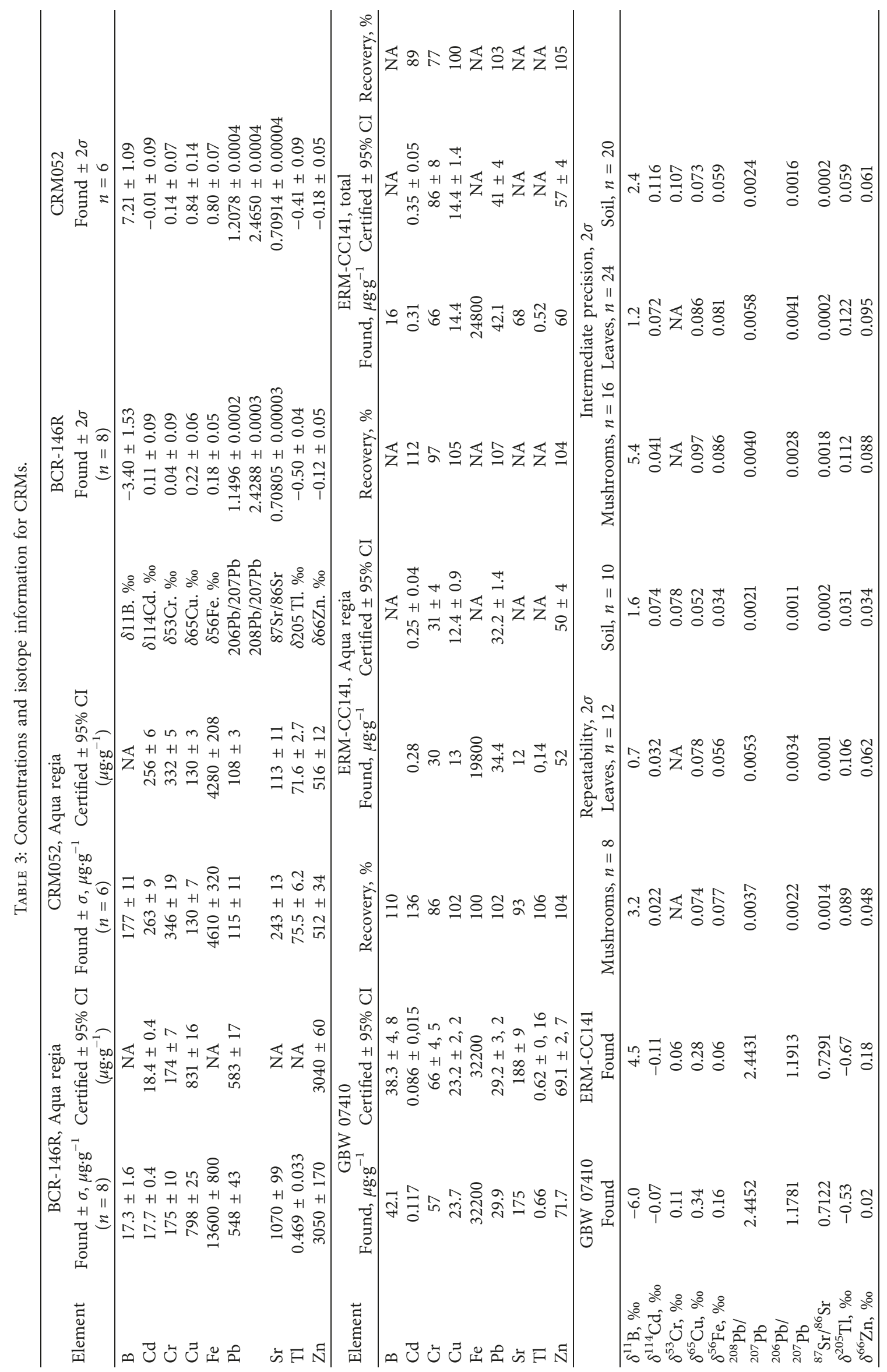


TABLE 4: Boron concentrations and isotopic composition in environmental samples from Luleå.

\begin{tabular}{|c|c|c|c|c|c|c|c|c|c|c|c|c|c|c|}
\hline \multirow{2}{*}{$\begin{array}{l}\text { Soil profiles } \\
\text { Fractions }\end{array}$} & \multicolumn{7}{|c|}{ Concentration, $\mu \mathrm{g} \cdot \mathrm{g}^{-1} \mathrm{DW}$} & \multicolumn{7}{|c|}{$\delta^{11} \mathrm{~B}, \%$} \\
\hline & F1 & $\mathrm{F} 2$ & F3 & $\mathrm{F} 4$ & F5 & F6 & Total & $\mathrm{F} 1$ & $\mathrm{~F} 2$ & F3 & F4 & F5 & F6 & Total \\
\hline \multicolumn{15}{|l|}{ City $(n=2)$} \\
\hline $0-15 \mathrm{~cm}$ & 0.57 & 0.24 & 0.48 & 1.05 & 1.71 & 3.87 & 7.91 & 2 & -9 & -17 & -12 & -12 & -7 & -9 \\
\hline $16-30 \mathrm{~cm}$ & 0.21 & 0.16 & 0.15 & 0.35 & 0.62 & 2.88 & 4.38 & -3 & -11 & -16 & -13 & -10 & -19 & -16 \\
\hline $31-45 \mathrm{~cm}$ & 0.04 & 0.11 & 0.04 & 0.09 & 0.38 & 1.90 & 2.56 & -9 & -11 & -10 & -15 & -16 & -24 & -22 \\
\hline $46-60 \mathrm{~cm}$ & 0.04 & 0.15 & 0.02 & 0.06 & 0.34 & 1.86 & 2.47 & -4 & -6 & -7 & -15 & -12 & -23 & -21 \\
\hline \multicolumn{15}{|l|}{ Suburb $(n=4)$} \\
\hline $0-15 \mathrm{~cm}$ & 0.16 & 0.06 & 0.10 & 0.17 & 0.83 & 5.15 & 6.47 & -25 & -24 & -17 & -2 & -17 & -14 & -14 \\
\hline $16-30 \mathrm{~cm}$ & 0.11 & 0.06 & 0.06 & 0.10 & 0.60 & 5.01 & 5.94 & -7 & -13 & -8 & -11 & -15 & -2 & -4 \\
\hline $31-45 \mathrm{~cm}$ & 0.06 & 0.11 & 0.04 & 0.07 & 0.80 & 5.10 & 6.17 & -10 & -4 & -2 & 10 & -6 & -4 & -4 \\
\hline \multirow[t]{3}{*}{$46-60 \mathrm{~cm}$} & 0.05 & 0.09 & 0.04 & 0.05 & 0.58 & 4.75 & 5.56 & -6 & -2 & -5 & 12 & -14 & -8 & -9 \\
\hline & \multicolumn{5}{|c|}{$\begin{array}{c}\text { Concentration, } \mu \mathrm{g} \cdot \mathrm{g}^{-1} \mathrm{DW} \\
\left(\mu \mathrm{g} \cdot \mathrm{L}^{-1} \text { for waters }\right)\end{array}$} & \multicolumn{9}{|c|}{$\delta^{11} \mathrm{~B}, \%$} \\
\hline & Mean & Median & $\sigma$ & Min. & Max. & & & Mean & Median & $\sigma$ & Min. & Max. & Range & \\
\hline Top soils & 8 & 7 & 7 & 2 & 53 & & & -7 & -8 & 7 & -17 & 12 & 29 & \\
\hline Lysimetric waters & 10 & 7 & 12 & 5 & 54 & & & 4 & 4 & 3 & -2 & 7 & 9 & \\
\hline Mushroom & 1.3 & 0.6 & 1.5 & 0.2 & 6.8 & & & -8 & -10 & 13 & -24 & 3 & 27 & \\
\hline Litter & 21 & NA & NA & NA & NA & & & 8 & NA & NA & NA & NA & NA & \\
\hline Wood ash & 290 & NA & NA & NA & NA & & & 7 & NA & NA & NA & NA & NA & \\
\hline Needles & 14 & 13 & 6 & 6 & 31 & & & 17 & 19 & 9 & -2 & 38 & 40 & \\
\hline Leaves & 21 & 18 & 13 & 7 & 76 & & & 10 & 10 & 9 & -7 & 34 & 41 & \\
\hline Lichens & 2.7 & 2.4 & 1.1 & 1.6 & 6.6 & & & 32 & 37 & 9 & 13 & 40 & 27 & \\
\hline
\end{tabular}

TABLE 5: Cadmium concentrations and isotopic composition in environmental samples from Luleå.

\begin{tabular}{|c|c|c|c|c|c|c|c|c|c|c|c|c|c|c|}
\hline \multirow{2}{*}{$\begin{array}{l}\text { Soil profiles } \\
\text { Fractions }\end{array}$} & \multicolumn{7}{|c|}{ Concentration, $\mu \mathrm{g} \cdot \mathrm{g}^{-1} \mathrm{DW}$} & \multicolumn{7}{|c|}{$\delta^{114} \mathrm{Cd}, \%$} \\
\hline & F1 & $\mathrm{F} 2$ & F3 & $\mathrm{F} 4$ & F5 & F6 & Total & F1 & $\mathrm{F} 2$ & F3 & $\mathrm{F} 4$ & F5 & F6 & Total \\
\hline \multicolumn{15}{|l|}{ City $(n=2)$} \\
\hline $0-15 \mathrm{~cm}$ & 0.006 & 0.016 & 0.427 & 0.149 & 0.122 & 0.036 & 0.756 & 0.08 & 0.04 & 0.08 & -0.19 & -0.19 & - & -0.02 \\
\hline $16-30 \mathrm{~cm}$ & 0.003 & 0.012 & 0.137 & 0.056 & 0.049 & 0.032 & 0.289 & 0.17 & 0.40 & 0.09 & -0.69 & -0.31 & - & -0.10 \\
\hline $31-45 \mathrm{~cm}$ & 0.001 & 0.012 & 0.019 & 0.015 & 0.016 & 0.019 & 0.081 & 0.25 & 0.26 & -0.02 & -0.91 & -0.24 & - & -0.15 \\
\hline $46-60 \mathrm{~cm}$ & 0.001 & 0.016 & 0.005 & 0.010 & 0.015 & 0.017 & 0.065 & 0.26 & 0.33 & -0.06 & 0.00 & -0.91 & - & -0.09 \\
\hline \multicolumn{15}{|l|}{ Suburb $(n=4)$} \\
\hline $0-15 \mathrm{~cm}$ & 0.002 & 0.010 & 0.027 & 0.010 & 0.013 & 0.012 & 0.074 & 0.23 & - & - & - & - & - & \\
\hline $16-30 \mathrm{~cm}$ & 0.001 & 0.027 & 0.012 & 0.008 & 0.009 & 0.011 & 0.069 & - & - & - & - & - & - & \\
\hline $31-45 \mathrm{~cm}$ & 0.001 & 0.026 & 0.008 & 0.005 & 0.010 & 0.010 & 0.061 & - & - & - & - & - & - & \\
\hline \multirow[t]{3}{*}{$46-60 \mathrm{~cm}$} & 0.001 & 0.025 & 0.003 & 0.006 & 0.009 & 0.012 & 0.056 & 0.19 & - & - & - & - & - & \\
\hline & \multicolumn{5}{|c|}{$\begin{array}{c}\text { Concentration, } \mu \mathrm{g} \cdot \mathrm{g}^{-1} \mathrm{DW} \\
\left(\mu \mathrm{g} \cdot \mathrm{L}^{-1} \text { for waters }\right)\end{array}$} & \multicolumn{9}{|c|}{$\delta^{114} \mathrm{Cd}, \%$} \\
\hline & Mean & Median & $\sigma$ & Min. & Max. & & & Mean & Median & $\sigma$ & Min. & Max. & Range & \\
\hline Top soils & 0.66 & 0.51 & 0.32 & 0.13 & 2.40 & & & 0.08 & 0.09 & 0.14 & -0.32 & 0.42 & 0.74 & \\
\hline Lysimetric waters & 0.04 & 0.03 & 0.05 & 0.01 & 0.22 & & & 0.22 & 0.19 & 0.07 & 0.14 & 0.34 & 0.20 & \\
\hline Mushroom & 1.6 & 1.2 & 1.4 & 0.03 & 5.0 & & & 0.15 & 0.23 & 0.28 & -0.71 & 0.52 & 1.23 & \\
\hline Litter & 0.30 & NA & NA & NA & $\mathrm{NA}$ & & & 0.17 & NA & NA & NA & NA & NA & \\
\hline Wood ash & 3.3 & NA & NA & NA & NA & & & -0.33 & NA & NA & NA & NA & NA & \\
\hline Needles & 0.06 & 0.04 & 0.04 & 0.01 & 0.16 & & & 0.09 & 0.10 & 0.26 & -0.52 & 0.56 & 1.08 & \\
\hline Leaves & 0.33 & 0.29 & 0.16 & 0.06 & 0.83 & & & 0.46 & 0.48 & 0.29 & -0.60 & 1.31 & 1.91 & \\
\hline Lichens & 0.13 & 0.12 & 0.03 & 0.09 & 0.18 & & & 0.08 & 0.07 & 0.03 & 0.05 & 0.18 & 0.13 & \\
\hline
\end{tabular}

morphology at each location may play an important role in the differences observed. The final area of discussion will highlight a case study involving environmental pollution.

\subsubsection{Multielement Patterns}

(1) Batch-Type Leach Water and Lysimeritc Waters. The batch-type water leaches (fraction F1) and lysimetric waters have similar elemental concentrations, with the exception of $\mathrm{Cu}, \mathrm{Pb}$, and $\mathrm{Zn}$, (assuming an average water to soil mass ratio of 1:10 for the F1 fraction). The concentrations of the latter analytes are several orders of magnitude lower in lysimetric waters than in the F1 fraction. Most of the isotopic compositions of the elements follow a similar pattern. Isotopic ratios of radiogenic elements $(\mathrm{Pb}$ and $\mathrm{Sr}$ ) in the F1 fractions from upper soil layers overlap the compositions of lysimetric waters. The differences in the isotopic 
TABLE 6: Chromium concentrations and isotopic composition in environmental samples from Luleå.

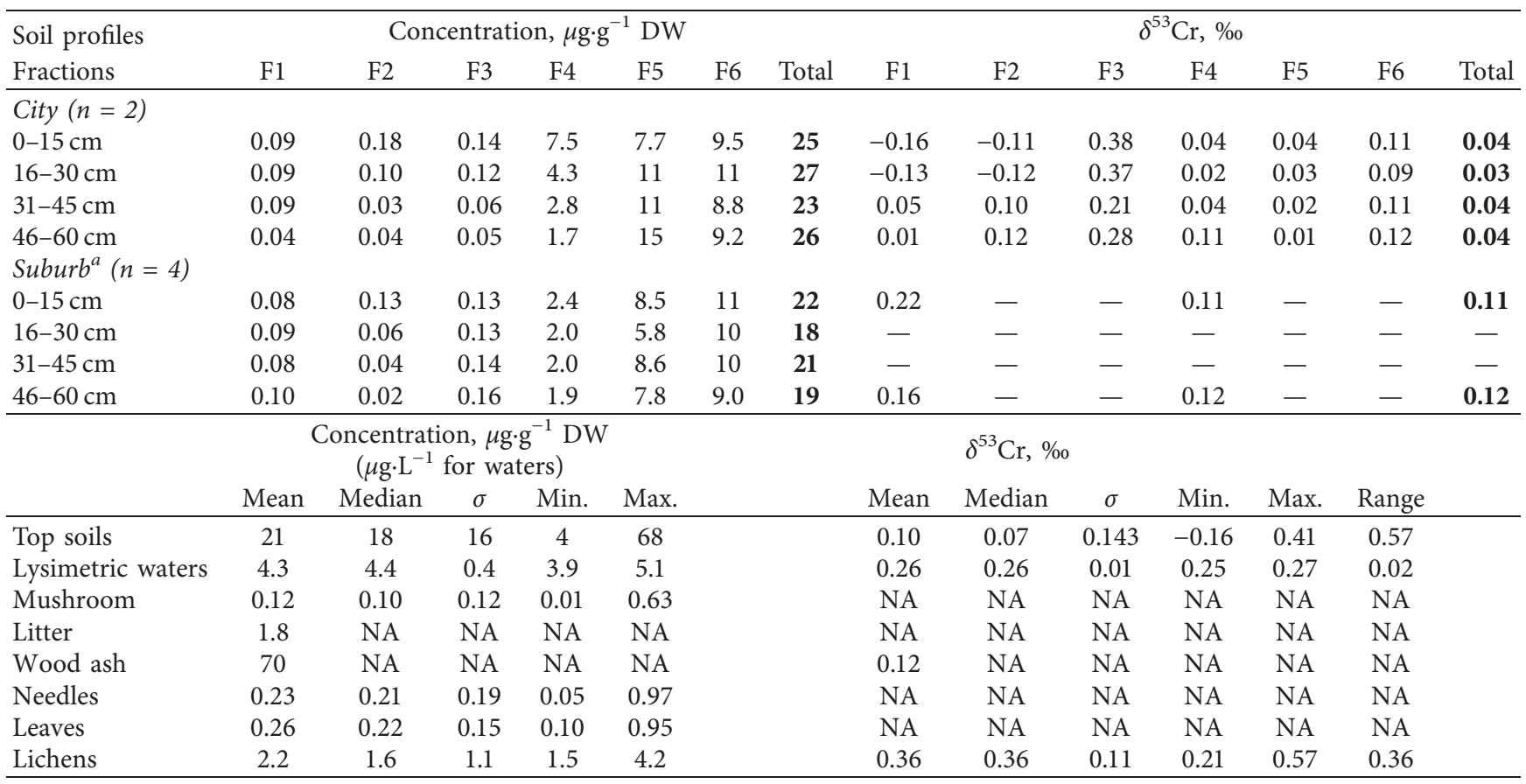

${ }^{a}$ Values reported as empty entries are due to pooling of several fractions. The isotopic values reported for the outmost top and bottom layers for F1 and F4 correspond to averaged values of respectively soil profiles $\mathrm{F} 1+\mathrm{F} 2+\mathrm{F} 3$ and $\mathrm{F} 4+\mathrm{F} 5+\mathrm{F} 6$ fractions.

TABLE 7: Copper concentrations and isotopic composition in environmental samples from Luleå.

\begin{tabular}{|c|c|c|c|c|c|c|c|c|c|c|c|c|c|c|}
\hline \multirow{2}{*}{$\begin{array}{l}\text { Soil profiles } \\
\text { Fractions }\end{array}$} & \multicolumn{7}{|c|}{ Concentration, $\mu \mathrm{g} \cdot \mathrm{g}^{-1} \mathrm{DW}$} & \multicolumn{7}{|c|}{$\delta^{65} \mathrm{Cu}, \%$} \\
\hline & F1 & $\mathrm{F} 2$ & F3 & $\mathrm{F} 4$ & F5 & F6 & Total & $\mathrm{F} 1$ & $\mathrm{~F} 2$ & F3 & $\mathrm{F} 4$ & F5 & F6 & Total \\
\hline \multicolumn{15}{|l|}{ City $(n=2)$} \\
\hline $0-15 \mathrm{~cm}$ & 0.33 & 0.58 & 0.08 & 26 & 11 & 1.2 & 39 & 0.10 & 0.05 & 0.38 & 0.23 & 0.29 & 0.06 & 0.26 \\
\hline $16-30 \mathrm{~cm}$ & 0.18 & 0.21 & 0.06 & 9.4 & 5.7 & 0.46 & 16 & 0.28 & 0.30 & 0.11 & 0.40 & 0.22 & -0.23 & 0.31 \\
\hline $31-45 \mathrm{~cm}$ & 0.16 & 0.11 & 0.03 & 3.1 & 3.5 & 0.31 & 7.2 & 0.15 & 0.30 & 0.92 & 0.16 & 0.25 & 0.51 & 0.26 \\
\hline $46-60 \mathrm{~cm}$ & 0.14 & 0.15 & 0.05 & 2.0 & 3.9 & 0.34 & 6.6 & 0.27 & 0.41 & 1.04 & 0.68 & 0.22 & -0.47 & 0.33 \\
\hline \multicolumn{15}{|l|}{ Suburb $(n=4)$} \\
\hline $0-15 \mathrm{~cm}$ & 0.13 & 0.50 & 0.06 & 2.1 & 3.0 & 0.58 & 6.4 & 0.35 & - & - & -0.14 & - & - & -0.06 \\
\hline $16-30 \mathrm{~cm}$ & 0.12 & 0.17 & 0.06 & 1.9 & 2.1 & 0.37 & 4.8 & - & - & - & - & - & - & - \\
\hline $31-45 \mathrm{~cm}$ & 0.09 & 0.07 & 0.07 & 1.5 & 2.6 & 0.58 & 4.9 & - & - & - & - & - & - & - \\
\hline \multirow[t]{3}{*}{$46-60 \mathrm{~cm}$} & 0.08 & 0.05 & 0.06 & 1.2 & 2.0 & 0.39 & 3.8 & 0.06 & - & - & -0.11 & - & - & -0.10 \\
\hline & \multicolumn{5}{|c|}{$\begin{array}{c}\text { Concentration, } \mu \mathrm{g} \cdot \mathrm{g}^{-1} \mathrm{DW} \\
\left(\mu \mathrm{g} \cdot \mathrm{L}^{-1} \text { for waters }\right)\end{array}$} & \multicolumn{9}{|c|}{$\delta^{65} \mathrm{Cu}, \%$} \\
\hline & Mean & Median & $\sigma$ & Min. & Max. & & & Mean & Median & $\sigma$ & Min. & Max. & Range & \\
\hline Top soils & 15 & 11 & 11 & 3 & 84 & & & 0.06 & 0.01 & 0.21 & -0.51 & 0.51 & 1.02 & \\
\hline Lysimetric waters & 0.6 & 0.5 & 0.2 & 0.4 & 1.1 & & & 0.76 & 0.72 & 0.17 & 0.64 & 0.99 & 0.35 & \\
\hline Mushroom & 44 & 39 & 25 & 10 & 120 & & & & -0.56 & 0.74 & -2.4 & 0.62 & 3.02 & \\
\hline Litter & 7 & NA & NA & NA & NA & & & -0.08 & NA & NA & NA & NA & NA & \\
\hline Wood ash & 180 & NA & NA & NA & NA & & & -1.00 & NA & NA & NA & NA & NA & \\
\hline Needles & 3.2 & 3.0 & 0.9 & 2.1 & 5.2 & & & -1.09 & -1.15 & 0.58 & -2.0 & 0.05 & 2.08 & \\
\hline Leaves & 7.1 & 5.3 & 3.4 & 3 & 17 & & & -0.35 & -0.33 & 0.35 & -1.34 & 0.83 & 2.15 & \\
\hline Lichens & 4.6 & 4.5 & 1.0 & 3.1 & 7.1 & & & 0.05 & 0.05 & 0.20 & -0.28 & 0.30 & 0.58 & \\
\hline
\end{tabular}

compositions of $\mathrm{B}, \mathrm{Cd}$, and Fe between these two matrices are also very minor. However, lysimetric waters were found to be preferentially enriched in the heavy isotopes of $\mathrm{Cr}, \mathrm{Cu}$, and $\mathrm{Zn}$ relative to the batch-type leach waters.

The overall isotopic and elemental pattern is most readily explained as the product of exchange between rainwater and soil particles. In natural systems, weakly sorbed elements such as $\mathrm{Cu}$ and $\mathrm{Pb}$ likely undergo reversible sorption and their retention is strongly depended on meteoric percolation dynamics.

At least for $\mathrm{Cu}$ and $\mathrm{Zn}$, this portioning/sorption process induces stable isotope fractionation [32]. 
TABLE 8: Iron concentrations and isotopic composition in environmental samples from Luleå.

\begin{tabular}{|c|c|c|c|c|c|c|c|c|c|c|c|c|c|c|}
\hline \multirow{2}{*}{$\begin{array}{l}\text { Soil profiles } \\
\text { Fractions }\end{array}$} & \multicolumn{7}{|c|}{ Concentration, $\mu \mathrm{g} \mathrm{g}^{-1} \mathrm{DW}$} & \multicolumn{7}{|c|}{$\delta^{56} \mathrm{Fe}, \% 0$} \\
\hline & F1 & $\mathrm{F} 2$ & F3 & $\mathrm{F} 4$ & F5 & F6 & Total & $\mathrm{F} 1$ & $\mathrm{~F} 2$ & F3 & $\mathrm{F} 4$ & F5 & F6 & Total \\
\hline \multicolumn{15}{|l|}{ City $(n=2)$} \\
\hline $0-15 \mathrm{~cm}$ & 26 & 110 & 420 & 2700 & 16000 & 6800 & 26000 & 0.21 & 0.21 & -2.0 & 0.33 & 0.22 & 0.17 & 0.19 \\
\hline $16-30 \mathrm{~cm}$ & 48 & 31 & 290 & 2100 & 14000 & 6000 & 22000 & 0.10 & 0.24 & -1.8 & 0.36 & 0.20 & 0.16 & 0.18 \\
\hline $31-45 \mathrm{~cm}$ & 75 & 15 & 200 & 740 & 12000 & 5300 & 18000 & 0.07 & -0.67 & -1.3 & 0.88 & 0.18 & 0.15 & 0.17 \\
\hline $46-60 \mathrm{~cm}$ & 39 & 17 & 160 & 330 & 14000 & 6000 & 21000 & 0.07 & -1.4 & -1.1 & 0.75 & 0.19 & 0.11 & 0.16 \\
\hline \multicolumn{15}{|l|}{ Suburb $(n=4)$} \\
\hline $0-15 \mathrm{~cm}$ & 45 & 66 & 560 & 530 & 7000 & 6100 & 14000 & -0.87 & - & - & 0.09 & - & - & 0.04 \\
\hline $16-30 \mathrm{~cm}$ & 73 & 33 & 620 & 780 & 5800 & 5100 & 13000 & - & - & - & - & - & - & - \\
\hline $31-45 \mathrm{~cm}$ & 54 & 10 & 630 & 550 & 7400 & 5500 & 14000 & - & - & - & - & - & - & - \\
\hline \multirow[t]{3}{*}{$46-60 \mathrm{~cm}$} & 54 & 7 & 580 & 460 & 7000 & 5400 & 13000 & -0.49 & - & - & 0.20 & - & - & 0.16 \\
\hline & \multicolumn{5}{|c|}{$\begin{array}{c}\text { Concentration, } \mu \mathrm{g} \cdot \mathrm{g}^{-1} \mathrm{DW} \\
\left(\mu \mathrm{g} \cdot \mathrm{L}^{-1} \text { for waters }\right)\end{array}$} & \multicolumn{9}{|c|}{$\delta^{56} \mathrm{Fe}, \%$} \\
\hline & Mean & Median & $\sigma$ & Min. & Max. & & & Mean & Median & $\sigma$ & Min. & Max. & Range & \\
\hline Top soils & 19000 & 17000 & 9000 & 4100 & 72000 & & & 0.07 & 0.10 & 0.13 & -0.33 & 0.45 & 0.78 & \\
\hline Lysimetric waters & 5200 & 5400 & 900 & 3600 & 5900 & & & 0.19 & 0.15 & 0.43 & -0.20 & 0.91 & 1.11 & \\
\hline Mushroom & 130 & 40 & 330 & 17 & 2100 & & & -0.48 & -0.47 & 0.35 & -1.16 & 0.26 & 1.42 & \\
\hline Litter & 1180 & NA & NA & NA & NA & & & 0.07 & NA & NA & NA & NA & NA & \\
\hline Wood ash & 3500 & NA & $\mathrm{NA}$ & NA & NA & & & 0.67 & NA & NA & NA & NA & NA & \\
\hline Needles & 140 & 110 & 110 & 33 & 440 & & & -0.41 & -0.35 & 0.41 & -1.4 & 0.05 & 1.45 & \\
\hline Leaves & 200 & 160 & 150 & 61 & 1500 & & & -0.29 & -0.26 & 0.20 & -0.84 & 0.09 & 0.93 & \\
\hline Lichens & 250 & 210 & 110 & 150 & 500 & & & -0.09 & -0.09 & 0.04 & -0.14 & -0.02 & 0.12 & \\
\hline
\end{tabular}

TABLE 9: Lead concentrations and isotopic composition in environmental samples from Luleå.

\begin{tabular}{|c|c|c|c|c|c|c|c|c|c|c|c|c|c|c|}
\hline \multirow{2}{*}{$\begin{array}{l}\text { Soil profiles } \\
\text { Fractions }\end{array}$} & \multicolumn{7}{|c|}{ Concentration, $\mu \mathrm{g} \cdot \mathrm{g}^{-1} \mathrm{DW}$} & \multicolumn{7}{|c|}{${ }^{206} \mathrm{~Pb} /{ }^{207} \mathrm{~Pb} /{ }^{208} \mathrm{~Pb} /{ }^{207} \mathrm{~Pb}$} \\
\hline & F1 & $\mathrm{F} 2$ & F3 & $\mathrm{F} 4$ & F5 & F6 & Total & F1 & $\mathrm{F} 2$ & F3 & $\mathrm{F} 4$ & F5 & F6 & Total \\
\hline \multicolumn{15}{|l|}{ City $(n=2)$} \\
\hline $0-15 \mathrm{~cm}$ & 0.31 & 0.02 & 3.2 & 52 & 30 & 5.9 & 91 & $1.14 / 2.37$ & $1.16 / 2.41$ & $1.15 / 2.39$ & $1.15 / 2.42$ & $1.17 / 2.43$ & $1.10 / 2.34$ & $1.15 / 2.41$ \\
\hline $16-30 \mathrm{~cm}$ & 0.17 & 0.02 & 0.75 & 9.4 & 18 & 6.6 & 35 & $1.17 / 2.43$ & $1.20 / 2.43$ & $1.16 / 2.43$ & $1.17 / 2.43$ & $1.20 / 2.44$ & $1.09 / 2.33$ & $1.17 / 2.41$ \\
\hline $31-45 \mathrm{~cm}$ & 0.08 & 0.02 & 0.27 & 1.3 & 7.7 & 4.3 & 14 & $1.28 / 2.50$ & $1.23 / 2.46$ & $1.22 / 2.49$ & $1.28 / 2.51$ & $1.35 / 2.49$ & $1.13 / 2.34$ & $1.27 / 2.44$ \\
\hline $46-60 \mathrm{~cm}$ & 0.02 & 0.03 & 0.08 & 0.15 & 4.3 & 4.9 & 9.4 & $1.42 / 2.61$ & $1.35 / 2.54$ & $1.42 / 2.60$ & $1.42 / 2.58$ & $1.54 / 2.62$ & $1.13 / 2.34$ & $1.32 / 2.47$ \\
\hline \multicolumn{15}{|l|}{$\begin{array}{l}\text { Suburb } \\
(n=4)\end{array}$} \\
\hline $0-15 \mathrm{~cm}$ & 0.04 & 0.04 & 0.54 & 1.2 & 5.9 & 5.9 & 14 & $1.32 / 2.57$ & $1.35 / 2.58$ & $1.29 / 2.54$ & $1.32 / 2.56$ & $1.38 / 2.55$ & $1.08 / 2.32$ & $1.24 / 2.44$ \\
\hline $16-30 \mathrm{~cm}$ & 0.04 & 0.08 & 0.34 & 0.26 & 4.6 & 5.5 & 11 & $1.30 / 2.55$ & $1.25 / 2.53$ & $1.26 / 2.48$ & $1.30 / 2.51$ & $1.37 / 2.55$ & $1.08 / 2.32$ & $1.22 / 2.43$ \\
\hline $31-45 \mathrm{~cm}$ & 0.04 & 0.08 & 0.19 & 0.52 & 4.2 & 6.8 & 12 & $1.33 / 2.56$ & $1.23 / 2.50$ & $1.29 / 2.52$ & $1.38 / 2.64$ & $1.42 / 2.58$ & $1.08 / 2.32$ & $1.21 / 2.42$ \\
\hline \multirow[t]{3}{*}{$46-60 \mathrm{~cm}$} & 0.02 & 0.07 & 0.07 & 0.05 & 3.2 & 7.4 & 11 & $1.44 / 2.64$ & $1.22 / 2.50$ & $1.46 / 2.61$ & $1.54 / 2.69$ & $1.55 / 2.64$ & $1.08 / 2.32$ & $1.21 / 2.41$ \\
\hline & \multicolumn{7}{|c|}{$\begin{array}{c}\text { Concentration, } \mu \mathrm{g} \cdot \mathrm{g}^{-1} \mathrm{DW}\left(\mu \mathrm{g} \cdot \mathrm{L}^{-1}\right. \\
\text { for waters) }\end{array}$} & \multicolumn{7}{|c|}{${ }^{206} \mathrm{~Pb} /{ }^{207} \mathrm{~Pb} /{ }^{208} \mathrm{~Pb} /{ }^{207} \mathrm{~Pb}$} \\
\hline & Mean & Median & $\sigma$ & Min. & Max. & & & Mean & Median & $\sigma$ & Min. & Max. & Range & \\
\hline Top soils & 18 & 16 & 21 & 5 & 470 & & & $1.35 / 2.49$ & $1.34 / 2.50$ & $0.15 / 0.01$ & $1.05 / 2.30$ & $1.77 / 2.61$ & $0.72 / 0.31$ & \\
\hline $\begin{array}{l}\text { Lysimetric } \\
\text { waters }\end{array}$ & 0.50 & 0.40 & 0.40 & 0.03 & 1.1 & & & $1.17 / 2.45$ & $1.17 / 2.44$ & $0.03 / 0.02$ & $1.10 / 2.42$ & $1.20 / 2.47$ & $0.10 / 0.05$ & \\
\hline Mushroom & 0.17 & 0.11 & 0.14 & 0.01 & 0.51 & & & $1.18 / 2.45$ & $1.17 / 2.45$ & $0.04 / 0.03$ & $1.10 / 2.41$ & $1.30 / 2.56$ & $0.20 / 0.15$ & \\
\hline Wood ash & 6.4 & NA & NA & NA & NA & & & $1.15 / 2.40$ & NA & NA & NA & NA & NA & \\
\hline Litter & 2.3 & NA & NA & NA & NA & & & $1.16 / 2.42$ & NA & NA & NA & NA & NA & \\
\hline Needles & 0.15 & 0.12 & 0.11 & 0.02 & 0.54 & & & $1.18 / 2.42$ & $1.18 / 2.42$ & $0.03 / 0.03$ & $1.11 / 2.34$ & $1.26 / 2.47$ & $0.15 / 0.21$ & \\
\hline Leaves & 0.29 & 0.21 & 0.29 & 0.04 & 2.1 & & & $1.18 / 2.44$ & $1.18 / 2.43$ & $0.03 / 0.02$ & $1.11 / 2.39$ & $1.25 / 2.49$ & $0.14 / 0.10$ & \\
\hline Lichens & 1.9 & 1.8 & 0.4 & 1.3 & 2.6 & & & $1.14 / 2.38$ & $1.14 / 2.38$ & $0.01 / 0.01$ & $1.14 / 2.37$ & $1.15 / 2.40$ & $0.01 / 0.03$ & \\
\hline
\end{tabular}

(2) Biologic Samples. Comparing the compositions of the biologic samples, i.e., lichen, mushrooms, and vascular plant tissue, from the same location can help differentiate biochemical variability from geochemical variability. Lichens do not contain roots, and therefore, their composition is expected to provide information on aerial (precipitation, sea aerosols, and long range pollution) sources of elements [33].
Unlike lichens, fruit bodies of mushrooms and vascular plant tissue are much less affected by direct contribution from airborne sources, both because of very short duration of exposure and significantly lower surface area/volume ratio.

For many elements ( $\mathrm{Cd}, \mathrm{Cu}, \mathrm{Fe}, \mathrm{Pb}$, and $\mathrm{Zn})$, lichens display a significantly narrower range of isotopic variability 
TABLE 10: Strontium concentrations and isotopic composition in environmental samples from Luleå.

\begin{tabular}{|c|c|c|c|c|c|c|c|c|c|c|c|c|c|c|}
\hline \multirow{2}{*}{$\begin{array}{l}\text { Soil profiles } \\
\text { Fractions }\end{array}$} & \multicolumn{7}{|c|}{ Concentration, $\mu \mathrm{g} \cdot \mathrm{g}^{-1} \mathrm{DW}$} & \multicolumn{7}{|c|}{${ }^{87} \mathrm{Sr} /{ }^{86} \mathrm{Sr}$} \\
\hline & $\mathrm{F} 1$ & F2 & F3 & $\mathrm{F} 4$ & F5 & F6 & Total & $\mathrm{F} 1$ & $\mathrm{~F} 2$ & F3 & F4 & F5 & F6 & Total \\
\hline \multicolumn{15}{|l|}{ City $(n=2)$} \\
\hline $0-15 \mathrm{~cm}$ & 0.5 & 2.3 & 11 & 5.4 & 6.4 & 141 & 167 & 0.730 & 0.734 & 0.734 & 0.733 & 0.732 & 0.727 & 0.727 \\
\hline $16-30 \mathrm{~cm}$ & 0.3 & 1.7 & 5.1 & 3.0 & 6.0 & 138 & 154 & 0.727 & 0.734 & 0.733 & 0.735 & 0.733 & 0.731 & 0.732 \\
\hline $31-45 \mathrm{~cm}$ & 0.1 & 1.2 & 1.0 & 0.70 & 7.0 & 161 & 171 & 0.724 & 0.734 & 0.728 & 0.726 & 0.735 & 0.727 & 0.727 \\
\hline $46-60 \mathrm{~cm}$ & 0.1 & 1.2 & 0.60 & 0.50 & 7.7 & 170 & 180 & 0.727 & 0.743 & 0.724 & 0.727 & 0.747 & 0.727 & 0.728 \\
\hline \multicolumn{15}{|l|}{ Suburb $(n=4)$} \\
\hline $0-15 \mathrm{~cm}$ & 0.3 & 0.6 & 1.2 & 0.62 & 6.5 & 146 & 146 & 0.727 & - & - & 0.732 & - & - & 0.732 \\
\hline $16-30 \mathrm{~cm}$ & 0.2 & 1.1 & 0.69 & 0.42 & 4.8 & 148 & 148 & - & - & - & - & - & - & - \\
\hline $31-45 \mathrm{~cm}$ & 0.1 & 1.5 & 0.41 & 0.33 & 6.0 & 179 & 179 & - & - & - & - & - & - & - \\
\hline \multirow[t]{3}{*}{$46-60 \mathrm{~cm}$} & 0.1 & 1.4 & 0.32 & 0.31 & 5.1 & 188 & 188 & 0.729 & - & - & 0.733 & - & - & 0.733 \\
\hline & \multicolumn{5}{|c|}{$\begin{array}{c}\text { Concentration, } \mu \mathrm{g} \cdot \mathrm{g}^{-1} \mathrm{DW} \\
\left(\mu \mathrm{g} \cdot \mathrm{L}^{-1} \text { for waters }\right)\end{array}$} & \multicolumn{9}{|c|}{${ }^{87} \mathrm{Sr} /{ }^{86} \mathrm{Sr}$} \\
\hline & Mean & Median & $\sigma$ & Min. & Max. & & & Mean & Median & $\sigma$ & Min. & Max. & Range & \\
\hline Top soils & 25 & 23 & 15 & 3 & 150 & & & 0.731 & 0.731 & 0.004 & 0.723 & 0.739 & 0.016 & \\
\hline Lysimetric waters & 12 & 10 & 2 & 8 & 12 & & & 0.733 & 0.733 & 0.001 & 0.732 & 0.733 & 0.010 & \\
\hline Mushroom & 0.5 & 0.3 & 0.2 & 0.1 & 1.8 & & & 0.729 & 0.727 & 0.006 & 0.721 & 0.750 & 0.039 & \\
\hline Litter & 28 & NA & NA & NA & NA & & & 0.731 & NA & NA & NA & NA & NA & \\
\hline Wood ash & 950 & NA & NA & NA & NA & & & 0.732 & NA & NA & NA & NA & NA & \\
\hline Needles & 18 & 15 & 16 & 2 & 63 & & & 0.734 & 0.733 & 0.007 & 0.724 & 0.754 & 0.030 & \\
\hline Leaves & 34 & 31 & 16 & 6 & 120 & & & 0.730 & 0.730 & 0.005 & 0.721 & 0.742 & 0.024 & \\
\hline Lichens & 8.3 & 7.4 & 3.9 & 2.5 & 16 & & & 0.733 & 0.731 & 0.006 & 0.725 & 0.742 & 0.017 & \\
\hline
\end{tabular}

TABLE 11: Thallium concentrations and isotopic composition in environmental samples from Luleå.

\begin{tabular}{|c|c|c|c|c|c|c|c|c|c|c|c|c|c|c|}
\hline \multirow{2}{*}{$\begin{array}{l}\text { Soil profiles } \\
\text { Fractions }\end{array}$} & \multicolumn{7}{|c|}{ Concentration, $\mu \mathrm{g} \cdot \mathrm{g}^{-1} \mathrm{DW}$} & \multicolumn{7}{|c|}{$\delta^{205} \mathrm{Tl}, \% \mathrm{o}$} \\
\hline & $\mathrm{F} 1$ & $\mathrm{~F} 2$ & F3 & F4 & F5 & F6 & Total & F1 & $\mathrm{F} 2$ & $\mathrm{~F} 3$ & $\mathrm{~F} 4$ & F5 & F6 & Total \\
\hline \multicolumn{15}{|l|}{ City $(n=2)$} \\
\hline $0-15 \mathrm{~cm}$ & 0.004 & 0.002 & 0.024 & 0.046 & 0.058 & 0.32 & 0.45 & 0.36 & 0.10 & -0.02 & -0.32 & -0.45 & -0.47 & -0.42 \\
\hline $16-30 \mathrm{~cm}$ & 0.001 & 0.001 & 0.009 & 0.024 & 0.070 & 0.40 & 0.50 & 0.36 & 0.12 & 0.00 & -0.39 & -0.40 & -0.49 & -0.46 \\
\hline $31-45 \mathrm{~cm}$ & 0.0003 & 0.0007 & 0.002 & 0.006 & 0.051 & 0.30 & 0.36 & 0.20 & -0.10 & -0.21 & -0.28 & -0.27 & -0.54 & -0.50 \\
\hline $46-60 \mathrm{~cm}$ & 0.0002 & 0.0006 & 0.002 & 0.004 & 0.064 & 0.31 & 0.39 & 0.18 & -0.07 & -0.26 & -0.38 & -0.28 & -0.57 & -0.52 \\
\hline \multicolumn{15}{|l|}{ Suburb $(n=4)$} \\
\hline $0-15 \mathrm{~cm}$ & 0.001 & 0.001 & 0.004 & 0.007 & 0.081 & 0.48 & 0.57 & -0.08 & - & - & -0.52 & - & - & -0.51 \\
\hline $16-30 \mathrm{~cm}$ & 0.001 & 0.0008 & 0.003 & 0.005 & 0.054 & 0.50 & 0.56 & - & - & - & - & - & - & - \\
\hline $31-45 \mathrm{~cm}$ & 0.0007 & 0.0007 & 0.002 & 0.004 & 0.074 & 0.53 & 0.62 & - & - & - & - & - & - & - \\
\hline \multirow[t]{3}{*}{$46-60 \mathrm{~cm}$} & 0.0005 & 0.0006 & 0.002 & 0.003 & 0.056 & 0.55 & 0.61 & -0.12 & - & - & -0.62 & - & - & -0.62 \\
\hline & \multicolumn{5}{|c|}{$\begin{array}{c}\text { Concentration, } \mu \mathrm{g} \cdot \mathrm{g}^{-1} \mathrm{DW} \\
\left(\mu \mathrm{g} \cdot \mathrm{L}^{-1} \text { for waters }\right)\end{array}$} & \multicolumn{9}{|c|}{$\delta^{205} \mathrm{Tl}, \%$} \\
\hline & Mean & Median & $\sigma$ & Min. & Max. & & & Mean & Median & $\sigma$ & Min. & Max. & Range & \\
\hline Top soils & 0.26 & 0.20 & 0.26 & 0.03 & 3.2 & & & -0.61 & -0.61 & 0.17 & -0.95 & -0.20 & 0.70 & \\
\hline Lysimetric waters & 0.04 & 0.04 & 0.024 & 0.002 & 0.07 & & & -0.14 & -0.17 & 0.07 & -0.22 & -0.05 & 0.17 & \\
\hline Mushroom & 0.015 & 0.009 & 0.016 & 0.002 & 0.081 & & & -0.18 & -0.28 & 0.30 & -0.62 & 0.42 & 1.04 & \\
\hline Litter & 0.030 & NA & NA & NA & NA & & & -0.41 & NA & NA & NA & NA & NA & \\
\hline Wood ash & 0.230 & NA & NA & NA & NA & & & -0.27 & NA & NA & NA & NA & NA & \\
\hline Needles & 0.010 & 0.010 & 0.020 & 0.001 & 0.090 & & & -0.31 & -0.38 & 0.21 & -0.63 & 0.16 & 0.89 & \\
\hline Leaves & 0.006 & 0.004 & 0.007 & 0.001 & 0.043 & & & -0.30 & -0.39 & 0.16 & -0.42 & -0.09 & 0.33 & \\
\hline Lichens & 0.023 & 0.009 & 0.024 & 0.004 & 0.060 & & & -0.53 & -0.54 & 0.10 & -0.72 & -0.41 & 0.31 & \\
\hline
\end{tabular}

than mushrooms, leaves, and needles. This is probably a reflection of the isotopically homogenous nature of Aeolian inputs combined with limited biologic fractionation of stable isotopes within lichen $[16,34,35]$. Lichens have significantly higher concentrations of $\mathrm{Cr}, \mathrm{Pb}$, and $\mathrm{Tl}$ and lower concentrations of $\mathrm{B}$ and $\mathrm{Sr}$ relative to leaves and needles. This indicates that atmospheric pollution is a significant source for heavy metals in the region relative to potential geologic sources.
The mushrooms are not only accumulating $\mathrm{Cd}, \mathrm{Cu}$, and $\mathrm{Zn}$ relative to the water-leachable element fraction of soils, but, with the exception of $\mathrm{Cd}$, the isotopic composition of these metals also differs between the mushrooms and lysimeric waters and/or the local soil. The most likely explanation of this isotopic fractionation is the active uptake of heavy metals by siderophilic root exudates. Lithophilic and chalcophilic elements ( $\mathrm{B}, \mathrm{Cd}, \mathrm{Pb}, \mathrm{Sr}$, and $\mathrm{Tl}$ ) do not appear to 
TABLE 12: Zinc concentrations and isotopic composition in environmental samples from Luleå.

\begin{tabular}{|c|c|c|c|c|c|c|c|c|c|c|c|c|c|c|}
\hline \multirow{2}{*}{$\begin{array}{l}\text { Soil profiles } \\
\text { Fractions }\end{array}$} & \multicolumn{7}{|c|}{ Concentration, $\mu \mathrm{g} \cdot \mathrm{g}^{-1} \mathrm{DW}$} & \multicolumn{7}{|c|}{$\delta^{66} \mathrm{Zn}, \%$} \\
\hline & $\mathrm{F} 1$ & $\mathrm{~F} 2$ & F3 & $\mathrm{F} 4$ & F5 & F6 & Total & F1 & $\mathrm{F} 2$ & F3 & $\mathrm{F} 4$ & F5 & F6 & Total \\
\hline \multicolumn{15}{|l|}{ City $(n=2)$} \\
\hline $0-15 \mathrm{~cm}$ & 7.2 & 230 & 180 & 96 & 38 & 13 & 560 & -0.13 & -0.07 & 0.01 & 0.02 & -0.24 & 0.30 & -0.04 \\
\hline $16-30 \mathrm{~cm}$ & 17 & 100 & 260 & 120 & 45 & 14 & 560 & -0.17 & -0.17 & -0.14 & -0.11 & -0.31 & 0.32 & -0.14 \\
\hline $31-45 \mathrm{~cm}$ & 5.4 & 49 & 35 & 18 & 23 & 11 & 140 & 0.01 & -0.07 & -0.09 & -0.06 & -0.01 & 0.34 & -0.01 \\
\hline $46-60 \mathrm{~cm}$ & 4.9 & 35 & 12 & 7.4 & 22 & 11 & 91 & 0.01 & 0.02 & 0.04 & 0.00 & 0.30 & 0.37 & 0.15 \\
\hline \multicolumn{15}{|l|}{ Suburb $(n=4)$} \\
\hline $0-15 \mathrm{~cm}$ & 7.9 & 75 & 27 & 5.6 & 15 & 12 & 140 & -0.23 & - & - & 0.11 & - & - & -0.09 \\
\hline $16-30 \mathrm{~cm}$ & 10 & 49 & 24 & 5.5 & 11 & 11 & 110 & - & - & - & - & - & - & - \\
\hline $31-45 \mathrm{~cm}$ & 7.6 & 110 & 13 & 3.4 & 14 & 10 & 160 & - & - & - & - & - & - & - \\
\hline \multirow[t]{3}{*}{$46-60 \mathrm{~cm}$} & 10 & 28 & 14 & 3.6 & 15 & 10 & 84 & -0.13 & - & - & 0.10 & - & - & 0.03 \\
\hline & \multicolumn{5}{|c|}{$\begin{array}{c}\text { Concentration, } \mu \mathrm{g} \cdot \mathrm{g}^{-1} \mathrm{DW} \\
\left(\mu \mathrm{g} \cdot \mathrm{L}^{-1} \text { for waters }\right)\end{array}$} & \multicolumn{9}{|c|}{$\delta^{66} \mathrm{Zn}, \%$} \\
\hline & Mean & Median & $\sigma$ & Min. & Max. & & & Mean & Median & $\sigma$ & Min. & Max. & Range & \\
\hline Top soils & 93 & 83 & 72 & 28 & 640 & & & 0.18 & 0.16 & 0.20 & -0.47 & 0.69 & 1.16 & \\
\hline Lysimetric waters & 5.0 & 4.6 & 3.2 & 1.3 & 11 & & & 0.15 & 0.14 & 0.13 & -0.05 & 0.30 & 0.35 & \\
\hline Mushroom & 120 & 110 & 50 & 40 & 260 & & & 0.58 & 0.55 & 0.36 & -0.16 & 1.25 & 1.41 & \\
\hline Litter & 220 & NA & NA & NA & NA & & & 0.00 & NA & NA & NA & NA & NA & \\
\hline Wood ash & 6200 & NA & NA & NA & NA & & & -0.45 & NA & NA & NA & NA & NA & \\
\hline Needles & 51 & 50 & 19 & 22 & 96 & & & -0.09 & -0.08 & 0.30 & -0.96 & 0.67 & 1.64 & \\
\hline Leaves & 220 & 180 & 140 & 11 & 800 & & & -0.13 & -0.11 & 0.21 & -0.94 & 0.24 & 1.18 & \\
\hline Lichens & 57 & 60 & 8 & 36 & 70 & & & 0.04 & 0.07 & 0.06 & -0.07 & 0.11 & 0.17 & \\
\hline
\end{tabular}

TABLE 13: Concentration and isotopic data for two contaminated soils (landfill A and landfill B) and waste samples from local industries (Fe, $\mathrm{Cu}$ slag, and fly ash).

\begin{tabular}{lcccccccccccc}
\hline & $\mathrm{Cd}, \mu \mathrm{g} \cdot \mathrm{g}^{-1}$ & $\delta^{114} \mathrm{Cd}$ & $\mathrm{Cr}, \mu \mathrm{g} \cdot \mathrm{g}^{-1}$ & $\delta^{53} \mathrm{Cr}$ & $\mathrm{Cu}, \mu \mathrm{g} \cdot \mathrm{g}^{-1}$ & $\delta^{63} \mathrm{Cu}$ & $\mathrm{Fe}, \%$ & $\delta^{56} \mathrm{Fe}$ & $\mathrm{Zn}, \mu \mathrm{g} \cdot \mathrm{g}^{-1}$ & $\delta^{66} \mathrm{Zn}$ & $\mathrm{Pb}, \mu \mathrm{g} \cdot \mathrm{g}^{-1}$ & ${ }^{206} \mathrm{~Pb} /{ }^{207} \mathrm{~Pb}$ \\
\hline Landfill A & 1.5 & 0.0 & 450 & 0.2 & 2700 & -1.0 & 15 & 0.4 & 1100 & 1.4 & 40 & 1.32 \\
Landfill B & 0.6 & -1.5 & 2600 & 0.1 & 170 & -1.4 & 10 & 1.0 & 180 & -0.2 & 170 & 1.17 \\
Fe slag & 0.2 & 0.1 & 4100 & 0.1 & 100 & 0.7 & 12 & 0.9 & 150 & -0.7 & 250 \\
Cu slag & 11 & -0.2 & 650 & 0.1 & 16000 & 0.1 & 35 & 0.4 & 12000 & 0.0 & 200 & 1.16 \\
Fly ash & 1.1 & 1.7 & 50 & 0.2 & 25 & 0.3 & 2.7 & 0.2 & 50 & 0.6 & 15 & 1.21 \\
\hline
\end{tabular}

be significantly fractionated by mushrooms. The mushrooms' ability to accumulate $\mathrm{Cd}$ without inducing isotopic fraction may indicate an alternative biochemical mechanism for element uptake in mushrooms.

It is difficult to identify any clear patterns in the leaf and needle data (Tables 4-12). This may be a result of the far greater biochemical complexities of higher plants causing a much wider spread of interspecies and intertree isotopic variability. The litter layer was sampled to study potential isotopic shift related to nutrient reabsorption back into tree during late stages of senescence [36]. However, preliminary analysis of $\mathrm{Fe}$ and $\mathrm{Pb}$ concentration data demonstrate that the litter had become significantly contaminated by soil particles. The isotope signature of leaves in late stage of development therefore overlapped with the isotopic composition of the top soils.

\subsubsection{Single-Element Patterns}

(1) Boron. Total B concentrations in the soil profile decrease with depth in the city samples, but not in the suburban samples (Table 4). Between $80 \%$ and $95 \%$ of the total B content was associated with the refractory phases (F5-F6) consistent with mineral bound $\mathrm{B}$ being the main source of $\mathrm{B}$ in the soils [37]. Secondary mineral formation is associated with the preferential incorporation of ${ }^{10} \mathrm{~B}$ into the secondary minerals [38] resulting in a heavier residual $\mathrm{B}$ composition in solution. This process appears to be the best explanation of the composition of the lysimetric waters in the present study ( $\delta^{11} \mathrm{~B}_{\text {water-soil }} \approx+11 \%$ o). The $\mathrm{B}$ isotopic composition of the mobile fraction trends towards a heavier signature in the upper soil layers, probably reflecting input from vegetation with plant biomass having distinctly heavier values $\left(\delta^{11} \mathrm{~B}_{\text {leaves}}\right.$-needles-soil $\approx+20 \%$ o)

There is a notable trend towards heavier $\mathrm{B}$ isotopic signatures in the direction soil < plants $<$ lichens with soil consistently characterized by negative $\delta$-values and plant materials always having positive $\delta^{11} \mathrm{~B}$. Boron is not thought to be strongly fractionated by biological activity [39]; thus, this trend can, at least partially, also be explained by source mixing. The isotopic composition of the lichens (mean value of $+32 \%$ for $\delta^{11} \mathrm{~B}$ ) will be affected by aerial marine aerosols with $\delta^{11} \mathrm{~B}$ of about $+40 \%$ [40], and the influence from this source could also explain the isotopic composition of leaves and needles in coastal regions. The total span of $\delta^{11} \mathrm{~B}$ values 
for our samples vary by around 64\% (Table 4), corresponding to almost half of the published span of $\delta^{11} \mathrm{~B}$ values [41]. More interestingly, the results for our bioindicator samples (mushrooms, needles, leaves, and lichens) show similar variability to coffee beans collected from vastly different geographic areas [8, 42] (Table 4). Overall, the B isotope systems appear to have characteristics which favor its use as an abiotic tracer of source mixing.

(2) Cadmium. The soil in the city locations contained significantly more $\mathrm{Cd}$ than the suburban location (Table 5). Such concentration difference is more pronounced in the surface soil layers compared to deeper profiles. For example, the upper most layers of the city soil profiles contain, on average, about 10 times the concentration found in the deepest inorganic layer. The major part of $\mathrm{Cd}$ is present in relatively mobile phases as about $70 \%$ of the total soil Cd was found in fractions (F1-F2-F3-F4). The reducible fraction (F3) contains especially high concentrations of Cd. This pattern suggests an anthropogenic source of Cd in the urban soil samples, but $\mathrm{Cd}$ isotopes do not appear a reliable means of identifying the pollution source.

Published variations of $\delta^{114} \mathrm{Cd}$ values in rock and mineral samples do not exceed $1 \%$, excluding the extreme fractionation values published for extraterrestrial material [43]. This relatively narrow fractionation span (corresponding to $0.25 \%$ o per mass unit) is small compared to light elements such as $\mathrm{Li}$ and $\mathrm{B}$, reflecting less relative mass difference between $\mathrm{Cd}$ isotopes. The mass balanced soil $\mathrm{Cd}$ pool has an isotopic composition equal within measurement uncertainty to $\delta^{114} \mathrm{Cd}=0 \%$ and the fractions with highest Cd concentrations do not have a distinct isotope signature. Cadmium isotopes do however appear to be influenced by biological activity.

There are limited published data on the $\mathrm{Cd}$ isotope ratios of nongeologic material, while geologic matrixes display a narrow range of isotope compositions. The range in $\delta^{114} \mathrm{Cd}$ values found in the leaves from the studied area (almost 2\%o, Table 5) by far exceeds the variability of the geologic samples and the soil and lysimetric waters in this study. Thus, significant $\mathrm{Cd}$ fractionation occurs during $\mathrm{Cd}$ uptake or incorporation into the leaves. Biologically mediated fractionation appears to be the best explanation of the heavier $\mathrm{Cd}$ isotopic composition in bioindicators $[2,21]$. It has been proposed [44] that carrier-mediated transport plays a major role in the root uptake process for Zn, and such process, also defined high-affinity transport, has been associated to a preferential translocation of heavier isotopes. Preferential incorporation of the heavy isotopes could explain also the observed shift between soil and leave isotope signatures (average $\delta^{114} \mathrm{Cd}_{\text {soil-leaves }} \approx$ $-0.4 \%$ ), as confirmed in recent studies [45], as well as the negative $\delta^{114} \mathrm{Cd}$ for the residual/immobile $\mathrm{Cd}$ pool in the soils.

(3) Chromium. There are minor differences in soil $\mathrm{Cr}$ concentrations between the sample locations (Table 6). Top soils have slightly lower $\mathrm{Cr}$ contents, which is probably due to dilution by $\mathrm{Cr}$-poor organic material (offal). The mobile fractions contain only a minor share of the total $\mathrm{Cr}$ pool irrespective of location or depth; on average, $83-88 \%$ of the total $\mathrm{Cr}$ is associated with residual phases. $\mathrm{Cr}$ isotopic compositions are very homogenous with practically all ratios in soils being the same within the uncertainty of the method (Table 3).

The Cr content of mushrooms, needles, and leaves was too low for accurate isotopic ratio measurements. $\delta^{53} \mathrm{Cr}$ values increased from soil-to-soil solution to lichens. Wet deposition could be the explanation for the heavy signature found for $\delta^{53} \mathrm{Cr}$ in lichens [46]. There is a positive correlation between $\mathrm{Cr}$ concentrations and increasing proximity to chromite processing steelworks and a negative correlation between $\delta^{53} \mathrm{Cr}$ values and increasing proximity to the steelworks implicating the smelter as the primary airborne Cr contributor in the area.

(4) Copper. Highest concentrations of $\mathrm{Cu}$ were found in the upper soil horizons from the city location and concentrations decrease with depth (Table 7). Similarly to $\mathrm{Cd}$ soil distribution, $\mathrm{Cu}$ is significantly less stratified in the suburb soil than in the soil from the city. However, $\mathrm{Cu}$ is bound in soils in a different pool than $\mathrm{Cd}$. Most of the $\mathrm{Cu}$ in top soil resides in the oxidizable fraction (F4), generally associated with organic matter. The smallest $\mathrm{Cu}$ pool was found within the F3 fraction in all depths and at all sample locations. Hence, $\mathrm{Cu}$ is not associated with $\mathrm{Mn}$ and Fe-oxides in the soil profiles.

The $\mathrm{Cu}$ isotopic ratios show no notable variations with soil depth. The $\mathrm{Cu}$ in the city soils has a heavy isotopic signature, while the suburban soil has a $\delta^{65} \mathrm{Cu}$ value of approximately $-0.1 \%$. This is consistent with previously reported findings for soil systems affected by $\mathrm{Cu}$ from an anthropogenic source [47], affirming that $\mathrm{Cu}$ isotopes can be used an indicator of anthropogenic pollution.

The biological samples have lighter $\mathrm{Cu}$ isotopic composition relative to the soil and lysimetric waters (Table 7). A shift towards lighter $\delta^{65} \mathrm{Cu}$ in vegetation is consistent with the previous reports of $\mathrm{Cu}$ fractionation in plants (e.g., [48]), and a similar general pattern was reported for other nutrients [21, 49]. However, the fractionation may not be a sole result of a biomediated reaction. While reduction in the rhizosphere by organic exudates is associated with the fractionation of the bioavailable pools of $\mathrm{Fe}$ and $\mathrm{Cu}$ towards lighter values, a biotic reduction has the same effect at least for $\mathrm{Fe}[50]$.

(5) Iron. The total concentrations of Fe in city soil samples are approximately twice than those found in the suburbs (Table 8). However, unlike $\mathrm{Cu}$ and $\mathrm{Cd}$, Fe concentrations do not change significantly with depth, and the majority of Fe was bound on the F5 and F6 fractions thus associated with primary bedrock mineral phases. Fe is one of the least mobile elements during chemical weathering creating the potential for soil iron concentrations to increase with increasing weathering intensity. This is consistent with the elevated Fe concentrations in the city soil probably reflecting a higher degree of chemical weathering, caused by a longer exposure, than the suburban soils. The iron isotopic composition of the 
city soil with the average $\delta^{56} \mathrm{Fe}$ value $=0.16 \%$ is within the range of values reported for granite reference materials $(0.13$ $\pm 0.12 \%$, [51]).

Clear iron isotopic variability within soil fractions is consistent with chemical weathering being the dominant driver of iron cycling in the soil. Iron isotopic composition of the F3 and F4 fractions resemble findings of previous studies $[52,53]$ attributing such variations to kinetic fractionation as a result of chemical weathering. The composition of leaching solutions used here for F1 and F2 soil fractions are different from those used in separation schemes designed specifically for Fe [54], which makes it difficult to compare the patterns. However, as mentioned in the previous section, redox processes may strongly fractionate iron $\left(\delta^{56} \mathrm{Fe}_{\mathrm{II}-\mathrm{III}}=-3 \%\right.$ [55]). It is possible that the $\mathrm{Fe}$ isotopic fractionation between the F1 and F2 fractions in the upper $30 \mathrm{~cm}$ and lower $30 \mathrm{~cm}$ reflects a soil redox change with the lower (deeper?) soils being enriched in $\mathrm{Fe}^{2+}$.

(6) Lead. Lead concentrations display the classic pattern expected for anthropogenic pollutant in soils. In deeper soil layers (depths $>30 \mathrm{~cm}$ ), $\mathrm{Pb}$ concentrations are constant at both locations. In the city location, there is a significant increase in $\mathrm{Pb}$ concentrations in the upper soil layers while $\mathrm{Pb}$ concentrations are constant at the surburb location. The excess $\mathrm{Pb}$ in the upper most city soil also has a significantly different $\mathrm{Pb}$ isotope composition than the geologic $\mathrm{Pb}$ in the region.

The isotopic composition of the $\mathrm{Pb}$ pool in the suburban samples is within the range of agricultural soils reported for northern Sweden [56]. The upper soil horizons from the city soil contain $\mathrm{Pb}$ with a significantly less radiogenic value. Back calculating the isotopic composition of this excess $\mathrm{Pb}$ (assuming end-member mixing) provides a value near that found in modern lichen ${ }^{206} \mathrm{~Pb} /{ }^{207} \mathrm{~Pb}=1.14$ and ${ }^{208} \mathrm{~Pb} /{ }^{207} \mathrm{~Pb}=$ 2.38 (Table 9) [57]. All the bioindicators studied here have a significantly less radiogenic isotopic composition than the presumed natural $\mathrm{Pb}$ baseline in deep soil horizons.

The absence of increased $\mathrm{Pb}$ in the suburban samples suggests that pollution is more local than what might be expected based on other locations in northern Sweden [58], and it might be explained by the relatively short time interval since the area emerged from the sea. The existence of various local pollution sources also appears to be evident in the wide spread of $\mathrm{Pb}$ isotopic ratios found in birch leaves and pine needles around Luleå.

Moving away from using $\mathrm{Pb}$ as a pollution tracer, the selective extractions also highlight that the influence preferential mineral weathering can have on the isotopic compositions of $\mathrm{Pb}$ pools within soils. The residual fraction F6 (HF digestion) of the SEP used in the present study contains a uniform $\mathrm{Pb}$ concentration at all locations and depths with significantly less radiogenic $\mathrm{Pb}$ isotopic composition than in other fractions (Table 9). Similarly, in a study of sediments from 31 lakes in Sweden [59], lower ${ }^{206} \mathrm{~Pb} /{ }^{207} \mathrm{~Pb}$ ratios were found in samples prepared using $\mathrm{HF}$ digestion compared to those treated using $\mathrm{HNO}_{3}+\mathrm{HClO}_{4}$ $(10: 1 \mathrm{v} / \mathrm{v})$. This was attributed to the $\mathrm{Pb}$ contribution from feldspars, which are more resistant to chemical weathering than most other minerals. In the present study, potassium feldspar is the main $\mathrm{Pb}$ carrier in the silicate soil fractions [60]. The majority of radiogenic $\mathrm{Pb}$, formed in silicates by ${ }^{238} \mathrm{U},{ }^{235} \mathrm{U}$, or ${ }^{232} \mathrm{Th}$ decay, might be efficiently leached in the first five SEP fractions. This leaves the final HF fractionation with a less radiogenic isotope composition reflecting those for the time of mineral formation approximately $2 \mathrm{Ga}$ years ago.

(7) Strontium. Total Sr concentrations display little variability across the dataset (Table 10). Top soils have slightly lower $\mathrm{Sr}$ concentrations, as for $\mathrm{Cr}$ most likely reflecting dilution effects by organic matter. The majority of $\mathrm{Sr}$ is found in the residual fractions (F5-F6), suggesting most of the element is bound in refractory mineral phases within the soil.

Mean ${ }^{87} \mathrm{Sr} /{ }^{86} \mathrm{Sr}$ ratios in all environmental matrices from Luleå fall into a relatively narrow range (0.729-0.734) with little differences in isotopic composition between soil and bioindicators. Thus, unlike B, Sr in needles, leaves, and lichens appears to be of a terrestrial rather than marine origin; seawater aerosols have less radiogenic $\mathrm{Sr}$ isotope ratios [61]. To a significant degree, the homogeneity of the ${ }^{87} \mathrm{Sr} /{ }^{86} \mathrm{Sr}$ ratios is expected given the composition of the local bedrock $[60,62]$.

Strontium isotopic ratios, primary variations in radiogenic ${ }^{87} \mathrm{Sr}$ abundance, have been extensively used for authentication and provenance studies [4]. Waters, crops, livestock, a variety of food products and ultimately man are known to reflect the Sr isotopic ratios typical for the geological setting in which they occur [63]. Strontium isotopic systematics has been also used, in combination with other systems, to trace anthropogenic sources in the environment $[10,11,64]$.

(8) Thallium. Total $\mathrm{Tl}$ concentrations in soils from city and suburban locations vary over a very limited range of approximately $0.4-0.6 \mu \mathrm{g} \cdot \mathrm{g}^{-1}$ at all depths (Table 11). Thallium predominantly resides in the residual fractions $(98 \%$ of $\mathrm{Tl}$ recovered in leachates F5-F6) most likely in the crystalline structure of silicate minerals such as potassium feldspar and quartz [65]. Concentration of the remaining $\mathrm{Tl}$ fractions, associated with reducible and oxidizable phases, is significantly higher in top soil from the city than that in the suburb. Our findings reaffirm previously reported $\mathrm{Tl}$ speciation data for rhizospheric soils [66].

There is very limited data on $\mathrm{Tl}$ isotopes in environmental samples if compared to other more "traditionally" studied isotopic systems. Tl isotopic ratio data from contaminated soils have been reported in literature with a $\delta^{205} \mathrm{Tl}$ value of $\approx-0.3 \%$ relative to the natural background, with average $\mathrm{Tl}$ concentrations of $0.75 \mu \mathrm{g} \cdot \mathrm{g}^{-1}$ [67]. The same study reported a lighter Tl signature with increasing soil depth in both the extractable and the residual fractions which is similar to our findings (Table 11).

$\mathrm{F} 1$ and F2 soil fractions have positive $\delta^{205} \mathrm{Tl}$ values. This heavier signature is then can be traced in significantly higher $\delta^{205} \mathrm{Tl}$ found in lysimetric waters, which in turn may contribute to the heavier $\mathrm{Tl}$ ratios found in mushrooms, needles, and leaves (Table 11). 
(9) Zinc. Zinc concentrations follow a similar pattern as for $\mathrm{Pb}$. The top soil layers from the city are significantly enriched in $\mathrm{Zn}$ while deeper soil horizons are characterized by more uniform concentrations (Table 12). The majority of $\mathrm{Zn}$ was found in the first four fractions (80-90\% leached in F1-F4), suggesting that source of anthropogenic $\mathrm{Zn}$, like $\mathrm{Pb}$, contains the element in a readily mobile form. Isotopic composition patterns for $\mathrm{Zn}$ and $\mathrm{Pb}$ show little similarities.

The deep soil layers $(46-60 \mathrm{~cm})$ have $\mathrm{Zn}$ isotopic compositions consistent with that of the local bedrock $(-0.05-$ 1.5\% ; converted from the "Lyon" JMC 3-0749 L standard) [47]. The soil horizons with the highest $\mathrm{Zn}$ concentrations seem to be slightly enriched in the light isotopes of $\mathrm{Zn}$, but the trend is not overly convincing. Previous studies reported that exogenous dust deposition does cause measurable shift in soils $\mathrm{Zn}$ isotopic composition [68] which otherwise is relatively constant [30] for less contaminated soils. This suggests that the source of anthropogenic $\mathrm{Zn}$ has a $\mathrm{Zn}$ isotope composition very close to the local bedrock. Previously, $\mathrm{Zn}$ fractionation in soil horizons was attributed to sorption onto the Fe oxyhydroxides [47].

The mean $\delta^{66} \mathrm{Zn}$ of top soils and lysimetric waters is approximately $+0.2 \%$, while a significantly higher value of $+0.6 \%$ was found in mushrooms indicating preferential uptake of heavier isotopes. The mechanism(s) of uptake of heavy metal ions by fungi can be via either an active or passive [69], and the prevailing heavier $\mathrm{Zn}$ signature in mushrooms compared to the soil pool suggests an active uptake strategy. On the contrary, $\mathrm{Zn}$ in needles and leaves is isotopically lighter most likely as a result of fractionation processes during $\mathrm{Zn}$ translocation through the shoot [70], favoring lighter isotopes.

3.2. Landfills and Industrial Wastes. Pollution source tracing using isotopic data can be challenging for a number of reasons:

(i) The composition of potential sources is seldom represented by isotopically well-defined end members

(ii) The isotopic signature of potential sources may overlap natural isotopic variability

(iii) Even where end-member compositions are distinct and well-constrained, there might be a large number of potential sources

(iv) For stable isotopes, numerous postdeposition fractionating processes may alter the original ratios (see, e.g., [4])

In order to demonstrate the severity of these complicating factors, concentration and isotopic data for waste samples from local industries ( $\mathrm{Cu}$ slag, Fe slag, and fly ash) and two heavily contaminated soils (landfills) were obtained (Table 13). These waste samples are very likely to be a major contributor to the landfills, and the goal of the exercise was to attempt identification of the presence of the industrial waste materials in the landfill using concentration patterns and isotopic fingerprints.
Both landfills are high in $\mathrm{Cd}$ with $\mathrm{Cd}$ concentrations at least 10 times higher than those found in the soil from the suburb location. The $\mathrm{Cd}$ in landfill $\mathrm{A}$ is relatively unfractionated which may point towards $\mathrm{Cu}$ slag as the most likely source of the element as $\mathrm{Cd}$ found in $\mathrm{Cu}$ slag has an isotopic composition undistinguishable from the mean for top soils. Fly ash, on the contrary, is significantly enriched in heavier $\mathrm{Cd}$ isotopes. Copper slag is also significantly enriched in $\mathrm{Cu}, \mathrm{Zn}$, and $\mathrm{Fe}$ relative to the other pollution sources so these elements would be expected to be enriched in landfill $\mathrm{A}$ if $\mathrm{Cu}$ slag was indeed the source of $\mathrm{Cd}$. This fits very well with the concentrations measurements of landfill A (Table 13). This raises the question of why the $\mathrm{Zn}$ and $\mathrm{Cu}$ isotopic compositions of landfill $\mathrm{A}$ are nothing like the $\mathrm{Zn}$ and $\mathrm{Cu}$ isotopic compositions of $\mathrm{Cu}$ slag.

Based on the overall elemental concentrations of $\mathrm{Zn}$ and $\mathrm{Cu}$ in landfill $\mathrm{A}$, it is hard to imagine that source mixing can account for the isotopic offset between the slag and the landfill. This is because it would take an enormous quantity of these elements from a highly fractionated source to attain the isotope composition of the landfill if there is really slag present in the landfill. Alternatively, post depositional processes might significantly alter the isotopic composition of landfill, though this contradicts by the observed lack of fractionation of $\mathrm{Fe}$ isotopes in landfill $\mathrm{A}$. The $\mathrm{Fe}$ isotope compositions of landfill $\mathrm{A}$ and $\mathrm{Cu}$ slag are identical, and most processes which heavily fractionate $\mathrm{Cu}$ and $\mathrm{Zn}$ isotopes would also be expected to fractionate Fe isotopes.

The picture is not clarified by the evaluation of landfill $\mathrm{B}$. Landfill $\mathrm{B}$ has a number of characteristics pointing towards Fe slag being the primary source of contamination with fly ash being a second significant source of contaminants. The $\mathrm{Fe}, \mathrm{Zn}, \mathrm{Cu}, \mathrm{Cr}$, and $\mathrm{Cd}$ concentrations and $\mathrm{Cr}, \mathrm{Fe}$, and $\mathrm{Zn}$ isotope compositions of landfill B are consistent with Fe slag mixed with some fly ash. At the same time, the $\mathrm{Cu}$ and $\mathrm{Cd}$ isotopic composition of landfill $\mathrm{B}$ requires an alternative explanation. It is possible that $\mathrm{Cu}$ isotopes are just not a reliable tracer of pollution sources in these landfills, but it is harder to account for $\mathrm{Cr}$ and $\mathrm{Zn}$ isotopes. The systems appear reliable in one of the landfills but not in the other.

Overall this case study highlights that pollution source mixing can be challenging, but the difficulty is proportional to the number of elements and isotopes measured. We cannot readily account for the behaviour of all the systems we measured, but a weight-of-evidence approach allows for the potential sources of pollution identified with some certainty. That is while any number of factors may complicate source tracing, they do not render the process total ineffective.

\section{Conclusions}

It is now technically feasible to rapidly analyze not only the trace metal composition of a broad range of the environmental samples but also the isotope compositions of all the metals. Expanding the number of elements utilized in isotope tracing provides a powerful way to decipher sources and fate of environmental exposure by adding degrees of freedom to the process. However, it also becomes 
increasingly complex to interpret large multielement data set. All the samples in this study were collected from spatially limited area, but a number of the analytes still displayed a significant range of concentration and isotopic values. As expected, the different elements also appear to be responding to different drivers such that for any given question, measuring more elements does not result in better/clearer answers. This highlights the need to simultaneously advance our understanding of single-element systems while progressing the technical capabilities required to measure more elements in more matrices. Natural variability, which often far exceeds the intermediate precision of the analytical method, needs to be considered when interpreting results of environmental studies.

\section{Data Availability}

The data used to support the findings of this study are included within the article.

\section{Disclosure}

This manuscript is based on Dr. Nicola Pallavicini's doctoral thesis entitled "Method development for isotope analysis of trace and Ultra-trace elements in environmental matrices".

\section{Conflicts of Interest}

The authors declare that there are no conflicts of interest regarding the publication of this paper.

\section{Supplementary Materials}

The supplementary material attached serves as a detailed and technical explanation of the analytical work behind the data provided in the manuscript. (Supplementary Materials)

\section{References}

[1] T. Arnold, T. Markovic, G. J. D. Kirk et al., "Iron and zinc isotope fractionation during uptake and translocation in rice (Oryza sativa) grown in oxic and anoxic soils," Comptes Rendus Geoscience, vol. 347, no. 7-8, pp. 397-404, 2015.

[2] I. Rodushkin, N. Pallavicini, E. Engström et al., "Assessment of the natural variability of $\mathrm{B}, \mathrm{Cd}, \mathrm{Cu}, \mathrm{Fe}, \mathrm{Pb}, \mathrm{Sr}, \mathrm{Tl}$ and $\mathrm{Zn}$ concentrations and isotopic compositions in leaves, needles and mushrooms using single sample digestion and twocolumn matrix separation," Journal of Analytical Atomic Spectrometry, vol. 31, no. 1, pp. 220-233, 2016.

[3] A. E. Shiel, D. Weis, and K. J. Orians, "Evaluation of zinc, cadmium and lead isotope fractionation during smelting and refining," Science of the Total Environment, vol. 408, no. 11, pp. 2357-2368, 2010.

[4] J. G. Wiederhold, "Metal stable isotope signatures as tracers in environmental geochemistry," Environmental Science and Technology, vol. 49, no. 5, pp. 2606-2624, 2015.

[5] D. F. Araújo, G. R. Boaventura, W. Machado et al., "Tracing of anthropogenic zinc sources in coastal environments using stable isotope composition," Chemical Geology, vol. 449, pp. 226-235, 2017.

[6] C. Kusonwiriyawong, M. Bigalke, F. Abgottspon et al., "Isotopic variation of dissolved and colloidal iron and copper in a carbonatic floodplain soil after experimental flooding," Chemical Geology, vol. 459, no. 13, pp. 13-23, 2017.

[7] Z. Liu, Z. Liu, X. Zhang, P. Xue, and H. Zhang, "RY10-4 suppressed metastasis of MDA-MB-231 by stabilizing ECM and E-cadherin," Biomedicine and Pharmacotherapy, vol. 68, no. 4, pp. 439-445, 2014.

[8] F. Serra, C. G. Guillou, F. Reniero et al., "Determination of the geographical origin of green coffee by principal component analysis of carbon, nitrogen and boron stable isotope ratios," Rapid Communications in Mass Spectrometry, vol. 19, no. 15, pp. 2111-2115, 2005.

[9] M. V. Baroni, N. S. Podio, R. G. Badini et al., "Linking soil, water, and honey composition to assess the geographical origin of argentinean honey by multielemental and isotopic analyses," Journal of Agricultural and Food Chemistry, vol. 63, no. 18, pp. 4638-4645, 2015.

[10] C. Hissler, P. Stille, A. Krein et al., "Identifying the origins of local atmospheric deposition in the steel industry basin of Luxembourg using the chemical and isotopic composition of the lichen Xanthoria parietina," Science of the Total Environment, vol. 405, no. 1-3, pp. 338-344, 2008.

[11] M. L. Geagea, P. Stille, F. Gauthier-Lafaye, and M. Millet, "Tracing of industrial aerosol sources in an urban environment using $\mathrm{Pb}, \mathrm{Sr}$, and $\mathrm{Nd}$ isotopes," Environmental Science \& Technology, vol. 42, no. 3, pp. 692-698, 2008.

[12] C. Cloquet, J. Carignan, G. Libourel, T. Sterckeman, and E. Perdrix, "Tracing source pollution in soils using cadmium and lead isotopes," Environmental Science and Technology, vol. 40, no. 8, pp. 2525-2530, 2006.

[13] H. Šillerová, V. Chrastný, M. Vítková et al., "Stable isotope tracing of $\mathrm{Ni}$ and $\mathrm{Cu}$ pollution in North-East Norway: potentials and drawbacks," Environmental Pollution, vol. 228, pp. 149-157, 2017.

[14] D. Widory, G. Vautour, and A. Poirier, "Atmospheric dispersion of trace metals between two smelters: an approach coupling lead, strontium and osmium isotopes from bioindicators," Ecological Indicators, vol. 84, pp. 497-506, 2018.

[15] T.-H.-B. Deng, C. Cloquet, Y.-T. Tang et al., "Nickel and zinc isotope fractionation in hyperaccumulating and nonaccumulating plants," Environmental Science and Technology, vol. 48, no. 20, pp. 11926-11933, 2014.

[16] D. Jouvin, D. J. Weiss, T. F. M. Mason et al., "Stable isotopes of $\mathrm{Cu}$ and $\mathrm{Zn}$ in higher plants: evidence for $\mathrm{Cu}$ reduction at the root surface and two conceptual models for isotopic fractionation processes," Environmental Science and Technology, vol. 46, no. 5, pp. 2652-2660, 2012.

[17] V. Chrastný, E. Čadková, A. Vaněk, L. Teper, J. Cabala, and M. Komárek, "Cadmium isotope fractionation within the soil profile complicates source identification in relation to $\mathrm{Pb}-\mathrm{Zn}$ mining and smelting processes," Chemical Geology, vol. 405, pp. 1-9, 2015.

[18] C. Cloquet, O. Rouxel, J. Carignan, and G. Libourel, "Natural cadmium isotopic variations in eight geological reference materials (NIST SRM 2711, BCR 176, GSS-1, GXR-1, GXR-2, GSD-12, nod-P-1, nod-A-1) and anthropogenic samples, measured by MC-ICP-MS," Geostandards and Geoanalytical Research, vol. 29, no. 1, pp. 95-106, 2005.

[19] C. N. Maréchal, P. Télouk, and F. Albarède, "Precise analysis of copper and zinc isotopic compositions by plasma-source mass spectrometry," Chemical Geology, vol. 156, no. 1-4, pp. 251-273, 1999.

[20] R. Gwiazda, D. Woolard, and D. Smith, "Improved lead isotope ratio measurements in environmental and biological samples with a double focussing magnetic sector inductively 
coupled plasma mass spectrometer (ICP-MS)," Journal of Analytical Atomic Spectrometry, vol. 13, no. 11, pp. 1233-1238, 1998.

[21] N. Pallavicini, E. Engström, D. C. Baxter, B. Öhlander, J. Ingri, and I. Rodushkin, "Cadmium isotope ratio measurements in environmental matrices by MC-ICP-MS," Journal of Analytical Atomic Spectrometry, vol. 29, no. 9, pp. 1570-1584, 2014.

[22] K. Tarricone, G. Wagner, and R. Klein, "Toward standardization of sample collection and preservation for the quality of results in biomonitoring with trees-a critical review," Ecological Indicators, vol. 57, pp. 341-359, 2015.

[23] B. Markert, "Multi-element analysis in ecosystems: basic conditions for representative sampling of plant materials," Fresenius' Zeitschrift für Analytische Chemie, vol. 335, no. 6, pp. 562-565, 1989.

[24] A. Bielicka-Giełdoń, E. Ryłko, and K. Żamojć, "Distribution, bioavailability and fractionation of metallic elements in allotment garden soils using the BCR sequential extraction procedure," Polish Journal of Environmental Studies, vol. 22, pp. 1013-1021, 2013.

[25] C. Lundberg and L. Peterson, "Land use history of central Lulea," Applied GIS, vol. 1, no. 3, pp. 1-25, 2005.

[26] M. Poutanen, "Land uplift at Kvarken archipelago and high coast UNESCO world heritage area," Holger Steffen, vol. 16, p. 16179, 2014.

[27] O. Mauer and E. Palátová, “The role of root system in silver birch (Betula pendula Roth) dieback in the air-polluted area of Krušné hory Mts," Stand, vol. 2003, pp. 191-199, 2003.

[28] G. Rauret, J. F. López-Sánchez, A. Sahuquillo et al., "Improvement of the BCR three step sequential extraction procedure prior to the certification of new sediment and soil reference materials," Journal of Environmental Monitoring, vol. 1, no. 1, pp. 57-61, 1999.

[29] D. C. Baxter, I. Rodushkin, E. Engström, and D. Malinovsky, "Revised exponential model for mass bias correction using an internal standard for isotope abundance ratio measurements by multi-collector inductively coupled plasma mass spectrometry," Journal of Analytical Atomic Spectrometry, vol. 21, no. 4, pp. 427-430, 2006.

[30] J. Viers, A. S. Prokushkin, O. S. Pokrovsky et al., "Zn isotope fractionation in a pristine larch forest on permafrostdominated soils in Central Siberia," Geochemical Transactions, vol. 16, no. 1, pp. 1-15, 2015.

[31] E. Engström, I. Rodushkin, J. Ingri et al., "Temporal isotopic variations of dissolved silicon in a pristine boreal river," Chemical Geology, vol. 271, no. 3-4, pp. 142-152, 2010.

[32] L. S. Balistrieri, D. M. Borrok, R. B. Wanty, and W. I. Ridley, "Fractionation of $\mathrm{Cu}$ and $\mathrm{Zn}$ isotopes during adsorption onto amorphous $\mathrm{Fe}(\mathrm{III})$ oxyhydroxide: experimental mixing of acid rock drainage and ambient river water," Geochimica et Cosmochimica Acta, vol. 72, no. 2, pp. 311-328, 2008.

[33] M. E. Conti and G. Cecchetti, "Biological monitoring: lichens as bioindicators of air pollution assessment-a review," Environmental Pollution, vol. 114, no. 3, pp. 471-492, 2001.

[34] M. Kiczka, J. G. Wiederhold, S. M. Kraemer, B. Bourdon, and R. Kretzschmar, "Iron isotope fractionation during Fe uptake and translocation in alpine plants," Environmental Science and Technology, vol. 44, no. 16, pp. 61446150, 2010.

[35] D. Houben, P. Sonnet, G. Tricot, N. Mattielli, E. Couder, and S. Opfergelt, "Impact of root-induced mobilization of zinc on stable $\mathrm{Zn}$ isotope variation in the soil-plant system,"
Environmental Science and Technology, vol. 48, no. 14, pp. 7866-7873, 2014.

[36] I. J. Wright and M. Westoby, "Nutrient concentration, resorption and lifespan: leaf traits of Australian sclerophyll species," Functional Ecology, vol. 17, no. 1, pp. 10-19, 2003.

[37] C. B. Tabelin, A. Hashimoto, T. Igarashi, and T. Yoneda, "Leaching of boron, arsenic and selenium from sedimentary rocks: II. $\mathrm{pH}$ dependence, speciation and mechanisms of release," Science of the Total Environment, vol. 473-474, pp. 244-253, 2014.

[38] P. Louvat, J. Hartman, T. Hosono et al., Behaviour of Boron Isotopes in the Streams and Springs of Aso Caldera, Kyushu, Japan, Goldschmidt Abstracts, Lyon, France, 2014.

[39] M. Rosner, W. Pritzkow, S. Voerkelius, and J. Vogl, Boron Isotopes-A New Tracer for the Origin and Authenticity of Food, Federal Institute for Materials Research and Testing, Berlin, Germany, 2009.

[40] G. L. Foster, P. A. E. Pogge Von Strandmann, and J. W. B. Rae, "Boron and magnesium isotopic composition of seawater," Geochemistry, Geophysics, Geosystems, vol. 11, no. 8, pp. 1-10, 2010.

[41] J. Xiao, Y. k. Xiao, Z. d. Jin, M. y. He, and C. q. Liu, "Boron isotope variations and its geochemical application in nature," Australian Journal of Earth Sciences, vol. 60, no. 4, pp. 431447, 2013.

[42] M. E. Wieser, S. S. Iyer, H. R. Krouse, and M. I. Cantagallo, "Variations in the boron isotope composition of Coffea arabica beans," Applied Geochemistry, vol. 16, no. 3, pp. 317-322, 2001.

[43] M. Rehkämper, F. Wombacher, T. J. Horner, and Z. Xue, "Natural and anthropogenic Cd isotope variations," in Handbook of Environmental Isotope Geochemistry, M. Baskaran, Ed., pp. 125-154, Springer, Berlin, Germany, 2012.

[44] D. J. Weiss, T. F. D. Mason, F. J. Zhao, G. J. D. Kirk, B. J. Coles, and M. S. A. Horstwood, "Isotopic discrimination of zinc in higher plants," New Phytologist, vol. 165, no. 3, pp. 703-710, 2005.

[45] M. Imseng, M. Wiggenhauser, A. Keller et al., "Fate of Cd in agricultural soils: a stable isotope approach to anthropogenic impact, soil formation, and soil-plant cycling," Environmental Science \& Technology, vol. 52, no. 4, pp. 1919-1928, 2018.

[46] S. Pontér, N. Pallavicini, E. Engström, D. C. Baxter, and I. Rodushkin, "Chromium isotope ratio measurements in environmental matrices by MC-ICP-MS," Journal of Analytical Atomic Spectrometry, vol. 31, no. 7, pp. 1464-1471, 2016.

[47] Z. Fekiacova, S. Cornu, and S. Pichat, "Tracing contamination sources in soils with $\mathrm{Cu}$ and $\mathrm{Zn}$ isotopic ratios," Science of the Total Environment, vol. 517, pp. 96-105, 2015.

[48] C. Weinstein, F. Moynier, K. Wang et al., "Isotopic fractionation of $\mathrm{Cu}$ in plants," Chemical Geology, vol. 286, no. 3-4, pp. 266-271, 2011.

[49] T. D. Bullen, "Stable isotopes of transition and post-transition metals as tracers in environmental studies," in Handbook of Environmental Isotope Geochemistry, M. Baskaran, Ed., pp. 177-203, Springer, Berlin, Germany, 2012.

[50] J. E. Roe, A. D. Anbar, and J. Barling, "Nonbiological fractionation of $\mathrm{Fe}$ isotopes: evidence of an equilibrium isotope effect," Chemical Geology, vol. 195, no. 1-4, pp. 69-85, 2003.

[51] J. B. Chapman, D. J. Weiss, Y. Shan, and M. Lemburger, "Iron isotope fractionation during leaching of granite and basalt by 
hydrochloric and oxalic acids," Geochimica et Cosmochimica Acta, vol. 73, no. 5, pp. 1312-1324, 2009.

[52] M. Kiczka, J. G. Wiederhold, J. Frommer et al., "Iron speciation and isotope fractionation during silicate weathering and soil formation in an alpine glacier forefield chronosequence," Geochimica et Cosmochimica Acta, vol. 75, no. 19, pp. 5559-5573, 2011.

[53] J. G. Wiederhold, S. M. Kraemer, N. Teutsch, P. M. Borer, A. N. Halliday, and R. Kretzschmar, "Iron isotope fractionation during proton-promoted, ligand-controlled, and reductive dissolution of goethite," Environmental Science \& Technology, vol. 40, no. 12, pp. 3787-3793, 2006.

[54] S. W. Poulton and D. E. Canfield, "Development of a sequential extraction procedure for iron: implications for iron partitioning in continentally derived particulates," Chemical Geology, vol. 214, no. 3-4, pp. 209-221, 2005.

[55] L. Wu, B. L. Beard, E. E. Roden, and C. M. Johnson, "Stable iron isotope fractionation between aqueous $\mathrm{Fe}(\mathrm{II})$ and hydrous ferric oxide," Environmental Science \& Technology, vol. 45, no. 5, pp. 1847-1852, 2011.

[56] C. Reimann, B. Flem, K. Fabian et al., "Lead and lead isotopes in agricultural soils of Europe-the continental perspective," Applied Geochemistry, vol. 27, no. 3, pp. 532-542, 2012.

[57] R. Bindler, I. Renberg, M. Brännvall, O. Emteryd, and F. ElDaoushy, "A whole-basin study of sediment accumulation using stable lead isotopes and flyash particles in an acidified lake, Sweden," Limnology and Oceanography, vol. 46, no. 1, pp. 178-188, 2001.

[58] J. Klaminder, R. Bindler, O. Emteryd, and I. Renberg, "Uptake and recycling of lead by boreal forest plants: quantitative estimates from a site in northern Sweden," Geochimica et Cosmochimica Acta, vol. 69, no. 10, pp. 2485-2496, 2005.

[59] I. Renberg, R. Bindler, and O. Emteryd, "Stable lead isotopes and lake sediments-a useful combination for the study of atmospheric lead pollution history," Science of the Total Environment, vol. 292, no. 1-2, pp. 45-54, 2002.

[60] C. Mellqvist, B. Ohlander, T. Skiold, and A. Wikstrom, "The Archaean-Proterozoic Palaeoboundary in the Luleå area, northern Sweden: field and isotope geochemical evidence for a sharp terrane boundary," Precambrian Research, vol. 96, no. 3-4, pp. 225-243, 1999.

[61] I. Rodushkin, T. Bergman, G. Douglas, E. Engström, D. Sörlin, and D. C. Baxter, "Authentication of Kalix (N.E. Sweden) vendace caviar using inductively coupled plasmabased analytical techniques: evaluation of different approaches," Analytica Chimica Acta, vol. 583, no. 2, pp. 310-318, 2007.

[62] F. Åberg and F. E. Wickman, "Variations of $87 \mathrm{Sr} / 86 \mathrm{Sr}$ in water from streams discharging into the Bothnian Bay, Baltic Sea," Hydrology Research, vol. 18, no. 1, pp. 33-42, 1987.

[63] B. Song, J. Ryu, H. S. Shin, and K. Lee, "Determination of the source of bioavailable Sr using $87 \mathrm{Sr} / 86 \mathrm{Sr}$ tracers: a case study of hot pepper and rice," Journal of Agricultural and Food Chemistry, vol. 62, no. 38, pp. 9232-9238, 2014.

[64] M. L. Geagea, P. Stille, F. Gauthier-Lafaye, T. Perrone, and D. Aubert, "Baseline determination of the atmospheric $\mathrm{Pb}, \mathrm{Sr}$ and $\mathrm{Nd}$ isotopic compositions in the Rhine valley, Vosges mountains (France) and the Central Swiss Alps," Applied Geochemistry, vol. 23, no. 6, pp. 1703-1714, 2008.

[65] A. Vaněk, T. Grygar, V. Chrastný, V. Tejnecký, P. Drahota, and M. Komárek, "Assessment of the BCR sequential extraction procedure for thallium fractionation using synthetic mineral mixtures," Journal of Hazardous Materials, vol. 176, no. 1-3, pp. 913-918, 2010.

[66] Y. Jia, T. Xiao, G. Zhou, and Z. Ning, "Thallium at the interface of soil and green cabbage (Brassica oleracea L. var. capitata L.): soil-plant transfer and influencing factors," Science of the Total Environment, vol. 450-451, pp. 140-147, 2013.

[67] M. Kersten, T. Xiao, K. Kreissig, A. Brett, B. J. Coles, and M. Rehkämper, "Tracing anthropogenic thallium in soil using stable isotope compositions," Environmental Science \& Technology, vol. 48, no. 16, pp. 9030-9036, 2014.

[68] M. Bigalke, S. Weyer, J. Kobza, and W. Wilcke, "Stable Cu and $\mathrm{Zn}$ isotope ratios as tracers of sources and transport of $\mathrm{Cu}$ and Zn in contaminated soil," Geochimica et Cosmochimica Acta, vol. 74, no. 23, pp. 6801-6813, 2010.

[69] A. Kapoor, T. Viraraghavan, and D. R. Cullimore, "Removal of heavy metals using the fungus Aspergillus niger," Bioresource Technology, vol. 70, no. 1, pp. 95-104, 1999.

[70] T. Fujii, F. Moynier, J. Blichert-Toft, and F. Albarède, "Density functional theory estimation of isotope fractionation of $\mathrm{Fe}, \mathrm{Ni}$, $\mathrm{Cu}$, and $\mathrm{Zn}$ among species relevant to geochemical and biological environments," Geochimica et Cosmochimica Acta, vol. 140, pp. 553-576, 2014. 

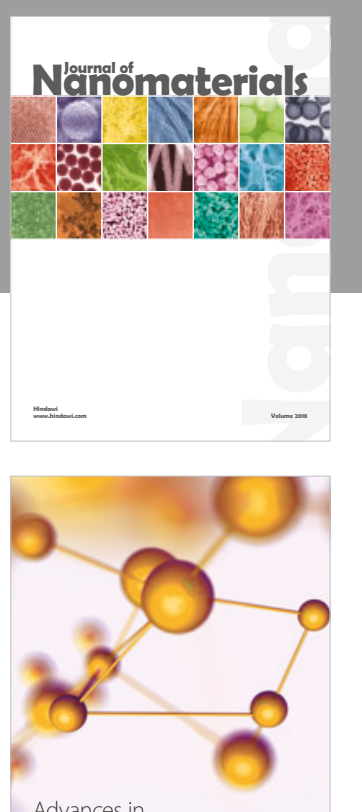

Physical Chemistry
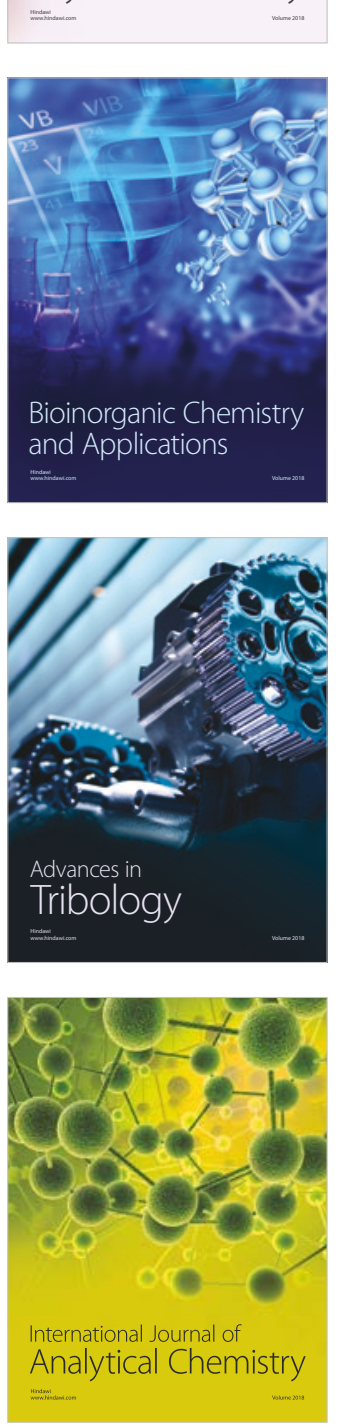

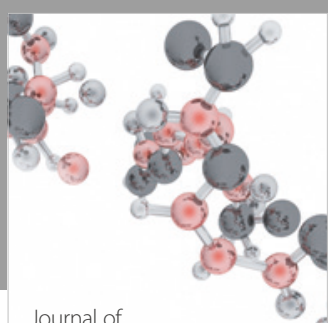

Analytical Methods

in Chemistry

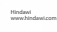

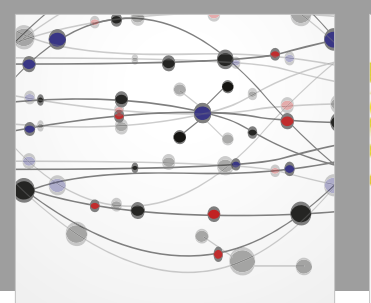

The Scientific World Journal

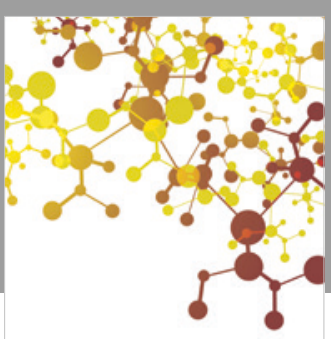

Journal of

Applied Chemistry
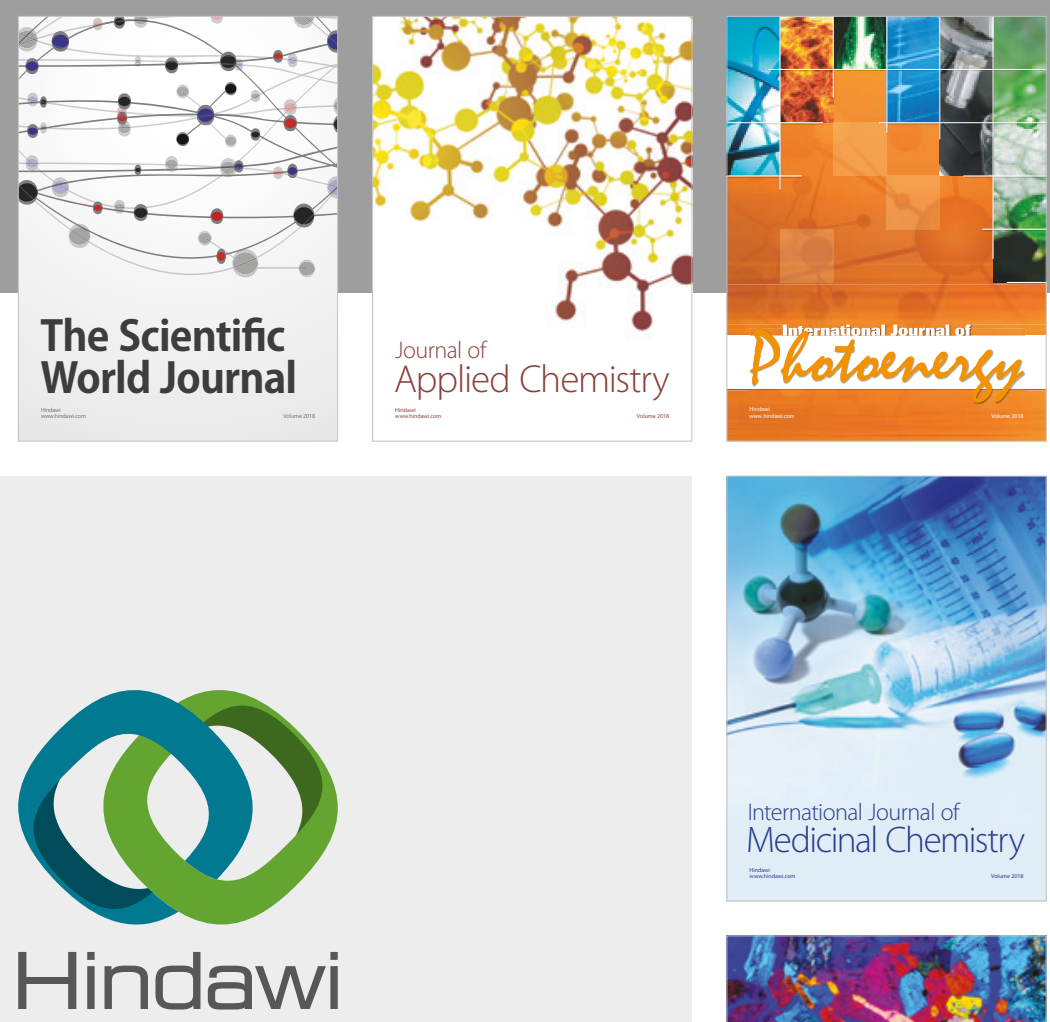

Submit your manuscripts at

www.hindawi.com
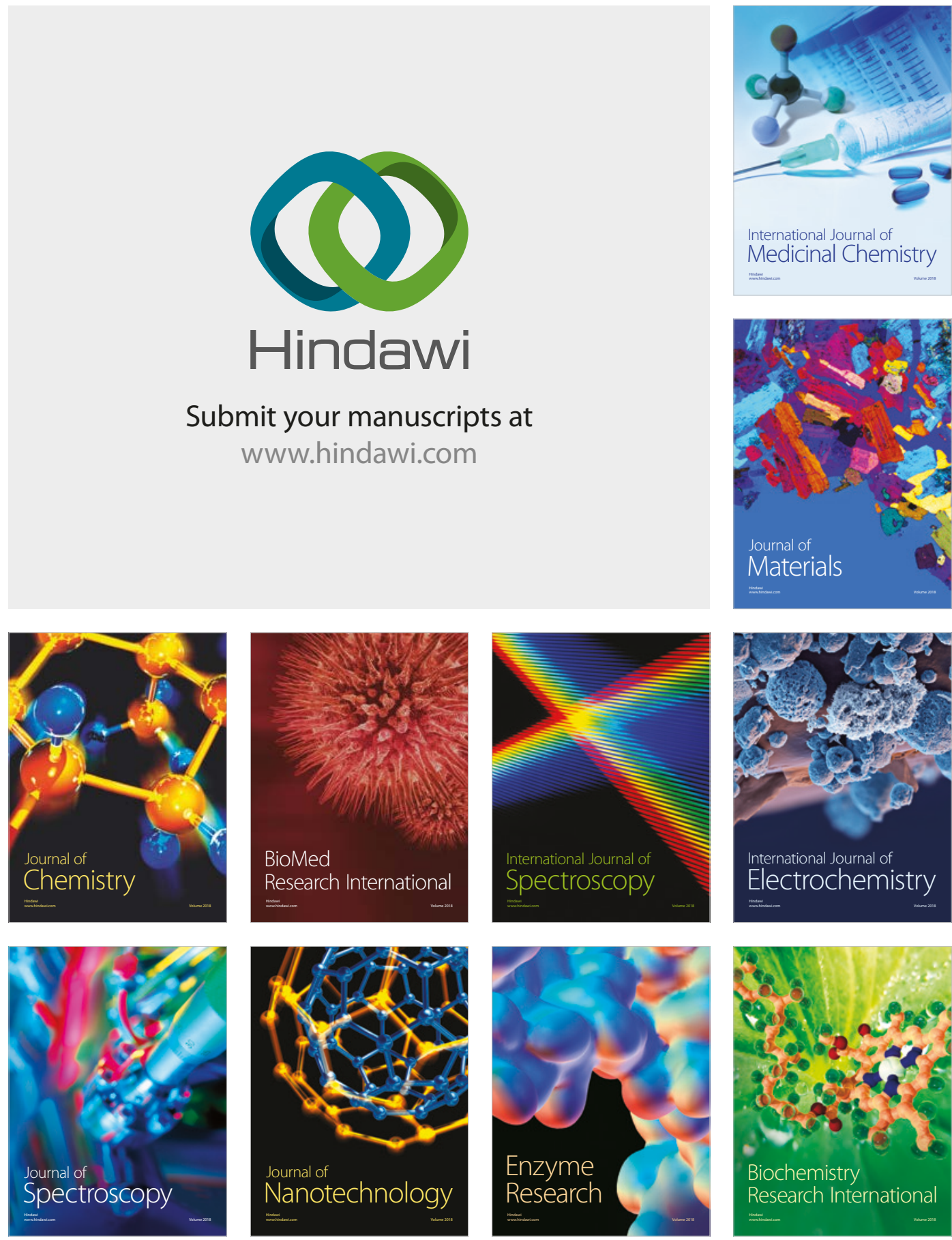
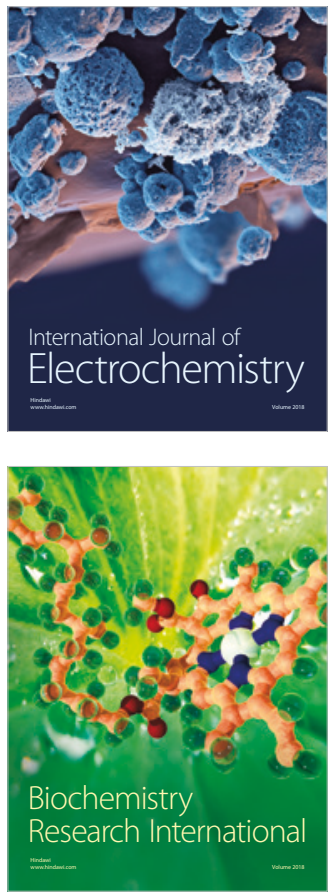